\title{
New fractional approaches for $n$-polynomial $P$-convexity with applications in special function theory
}

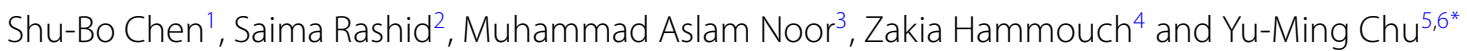

\author{
*Correspondence: \\ chuyuming2005@126.com \\ ${ }^{5}$ Department of Mathematics, \\ Huzhou University, Huzhou, China \\ ${ }^{6}$ Hunan Provincial Key Laboratory of \\ Mathematical Modeling and \\ Analysis in Engineering, Changsha \\ University of Science \& Technology, \\ Changsha, China \\ Full list of author information is \\ available at the end of the article
}

\begin{abstract}
Inequality theory provides a significant mechanism for managing symmetrical aspects in real-life circumstances. The renowned distinguishing feature of integral inequalities and fractional calculus has a solid possibility to regulate continuous issues with high proficiency. This manuscript contributes to a captivating association of fractional calculus, special functions and convex functions. The authors develop a novel approach for investigating a new class of convex functions which is known as an $n$-polynomial $\mathcal{P}$-convex function. Meanwhile, considering two identities via generalized fractional integrals, provide several generalizations of the Hermite-Hadamard and Ostrowski type inequalities by employing the better approaches of Hölder and power-mean inequalities. By this new strategy, using the concept of $n$-polynomial $\mathcal{P}$-convexity we can evaluate several other classes of $n$-polynomial harmonically convex, $n$-polynomial convex, classical harmonically convex and classical convex functions as particular cases. In order to investigate the efficiency and supremacy of the suggested scheme regarding the fractional calculus, special functions and $n$-polynomial $\mathcal{P}$-convexity, we present two applications for the modified Bessel function and $\mathfrak{q}$-digamma function. Finally, these outcomes can evaluate the possible symmetric roles of the criterion that express the real phenomena of the problem.
\end{abstract}

MSC: 26D15; 26D10; 90C23

Keywords: Hermite-Hadamard inequality; Generalized fractional integral; Ostrowski inequality; Hölder-işcan inequality; Improved-power-mean inequality; Modified Bessel function; $\mathfrak{q}$-digamma function

\section{Springer}

\section{Introduction}

Over the most recent couple of decades, fractional calculus [1-14] has been effectively utilized in modeling for a wide range of processes and systems in the field of engineering and applied sciences. The comprehensive applications in real-world problems are described by fractional operators including fluid mechanics such as exothermic chemical reactions or autocatalytic reactions, and it has been found to be an effective tool in interpretation and modeling of numerous problems appear in physics and applied mathematics [15]. Fractional integrodifferential equations contain derivatives of any complex or real order, being

(c) The Author(s) 2020. This article is licensed under a Creative Commons Attribution 4.0 International License, which permits use, sharing, adaptation, distribution and reproduction in any medium or format, as long as you give appropriate credit to the original author(s) and the source, provide a link to the Creative Commons licence, and indicate if changes were made. The images or other third party material in this article are included in the article's Creative Commons licence, unless indicated otherwise in a credit line to the material. If material is not included in the article's Creative Commons licence and your intended use is not permitted by statutory regulation or exceeds the permitted use, you will need to obtain permission directly from the copyright holder. To view a copy of this licence, visit http://creativecommons.org/licenses/by/4.0/. 
considered as a general form of differential equations. Numerous investigations have been proposed to improve the modeling accuracy in depicting the anomalous diffusion process, modeling different sorts of viscoelastic damping, precisely catching power-law frequency dependence and stimulating the flow of a fractional Maxwell fluid.

The fractional integral inequality [16-24] is one of the most popular approaches which is widely employed in many practical problems, optimization theory, engineering and technology. Integral inequalities of fractional strategies show substantially more ordinarily in several research areas and technology applications. The significant extent of utilities of the integral inequalities on convexity for both derivation and integration has been a subject of hot debate. Set et al. [25] illustrated numerous novel versions of Hermite-HadamardFejér type inequalities via fractional integral operators.

The main subject of this paper is the introduction of the notion of $n$-polynomial $\mathcal{P}$ convex functions and establishing the Hermite-Hadamard and Ostrowski type inequalities for $n$-polynomial $\mathcal{P}$-convex functions. In the deterministic case, most of the presented results are the refinements of the existing results for harmonically convex and classical convex functions in the relative literature. The new methodology is useful to explore the Mandelbrot and Julia sets for quadratic and cubic polynomials by introducing the term $s$ in $n$-polynomial convexity.

Now, we recall the Hermite-Hadamard inequality as follows:

Let $\hbar: I \rightarrow \mathbb{R}$ be a convex function. Then the double inequality

$$
\hbar\left(\frac{l_{1}+l_{2}}{2}\right) \leq\left(l_{2}-l_{1}\right)^{-1} \int_{l_{1}}^{l_{2}} \hbar(z) d z \leq \frac{\hbar\left(l_{1}\right)+\hbar\left(l_{2}\right)}{2}
$$

holds for all $l_{1}, l_{2} \in I$ with $l_{1} \neq l_{2}$.

Recently, the refinements, generalizations, extensions and variants for the HermitHadamard inequality have attracted the attention of many researchers.

In 1928, Ostrowski [26] established the Ostrowski inequality, which gives an upper bound for the approximation of the integral average $\left(l_{2}-l_{1}\right)^{-1} \int_{l_{1}}^{l_{2}} \hbar(\xi) d \xi$ by the value $\hbar(z)$ at $z \in\left[l_{1}, l_{2}\right]$, it can be stated as follows:

Let $\hbar:\left[l_{1}, l_{2}\right] \rightarrow \mathbb{R}$ be a differentiable mapping on $\left(l_{1}, l_{2}\right)$ such that $\left|\hbar^{\prime}(x)\right| \leq \mathcal{M}$ for all $z \in\left(l_{1}, l_{2}\right)$. Then the inequality

$$
\left|\hbar(z)-\left(l_{2}-l_{1}\right)^{-1} \int_{l_{1}}^{l_{2}} \hbar(\xi) d \xi\right| \leq \mathcal{M}\left(l_{2}-l_{1}\right)\left[\frac{1}{4}+\frac{\left(z-\frac{l_{1}+l_{2}}{2}\right)^{2}}{\left(l_{2}-l_{1}\right)^{2}}\right]
$$

holds for all $z \in\left(l_{1}, l_{2}\right)$ with the best possible constant $1 / 4$.

The Ostrowski inequality (1.2) plays a prominent role in pure and applied mathematics, particularly in approximation theory. In recent decades, many mathematicians have studied the Ostrowski inequality from the perspective of fractional calculus and convex analysis.

İşcan [27] presented a new form of the Hölder integral inequality as follows.

Theorem 1.1 (See [27]) Let $\alpha, \beta>1$ with $\alpha+\beta=\alpha \beta$, and $\hbar_{1}$ and $\hbar_{2}$ be two integrable real-valued functions defined on $\left[l_{1}, l_{2}\right]$ such that $\left|\hbar_{1}\right|^{\alpha}$ and $\left|\hbar_{2}\right|^{\beta}$ are integrable on $\left[l_{1}, l_{2}\right]$. 
Then one has

$$
\begin{aligned}
\int_{l_{1}}^{l_{2}}\left|\hbar_{1}(z) \hbar_{2}(z)\right| d z & \\
\leq & \frac{1}{l_{2}-l_{1}}\left[\left(\int_{l_{1}}^{l_{2}}\left(l_{2}-z\right)\left|\hbar_{1}(z)\right|^{\alpha} d z\right)^{1 / \alpha}\left(\int_{l_{1}}^{l_{2}}\left(l_{2}-z\right)\left|\hbar_{2}(z)\right|^{\beta} d z\right)^{1 / \beta}\right. \\
& \left.\quad+\left(\int_{l_{1}}^{l_{2}}\left(z-l_{1}\right)\left|\hbar_{1}(z)\right|^{\alpha} d z\right)^{1 / \alpha}\left(\int_{l_{1}}^{l_{2}}\left(z-l_{1}\right)\left|\hbar_{2}(z)\right|^{\beta} d z\right)^{1 / \beta}\right] .
\end{aligned}
$$

Kadakal et al. [28] generalized the Hölder-İsscan integral inequality (1.3) to the following form.

Theorem 1.2 (See [28]) Let $\alpha, \beta>1$ with $\alpha+\beta=\alpha \beta$, and $\hbar_{1}$ and $\hbar_{2}$ be two integrable real-valued functions defined on $\left[l_{1}, l_{2}\right]$ such that $\left|\hbar_{1}\right|^{\alpha}$ and $\left|\hbar_{2}\right|^{\beta}$ are integrable on $\left[l_{1}, l_{2}\right]$. Then

$$
\begin{aligned}
\int_{l_{1}}^{l_{2}}\left|\hbar_{1}(z) \hbar_{2}(z)\right| d z & \\
\leq & \frac{1}{l_{2}-l_{1}}\left[\left(\int_{l_{1}}^{l_{2}}\left(l_{2}-z\right)\left|\hbar_{1}(z)\right|^{\alpha} d z\right)^{1-1 / \alpha}\left(\int_{l_{1}}^{l_{2}}\left(l_{2}-z\right)\left|\hbar_{1}(z)\right|\left|\hbar_{2}(z)\right|^{\beta} d z\right)^{1 / \beta}\right. \\
& \left.\quad+\left(\int_{l_{1}}^{l_{2}}\left(z-l_{1}\right)\left|\hbar_{1}(z)\right|^{\alpha} d z\right)^{1-1 / \alpha}\left(\int_{l_{1}}^{l_{2}}\left(z-l_{1}\right)\left|\hbar_{1}(z)\right|\left|\hbar_{2}(z)\right|^{\beta} d z\right)^{1 / \beta}\right] .
\end{aligned}
$$

The inspiration of this work resonates in every part of this article. This paper has multiple purposes. Our first intention is to introduce the notion of the $n$-polynomial $\mathcal{P}$-convex function. Taking into account two fractional identities, we derived several Hermite-Hadamard type inequalities for the well-known integral inequalities such as weighted arithmetic-geometric mean, Young's inequality, improved power-mean and Hölder-İscan inequality. The second vital intention is to assemble the consequences from our findings for special functions such as hypergeometric functions, modified Bessel functions and $\mathfrak{q}$-digamma function. Our work's results are valuable in the generation of fractals utilizing an iterative methodology, which is a fascinating field of examination and has utilities in the improvement of geometrically aided design.

The paper is organized as follows. In Sect. 2, we recall some basic and fundamental definitions and lemmas. In Sect. 3, we provide the Hermite-Hadamard type inequality for $n$-polynomial $\mathcal{P}$-convex functions. In Sect. 4 , we establish the Hermite-Hadamard type inequalities for differentiable functions. In Sect. 5, we present the Ostrowski type inequalities for the aforesaid technique. In Sect. 6, we give the applications of our results.

\section{Preliminaries}

We first give some basic and novel definitions for various convex functions and generalized fractional integrals. 
Definition 2.1 (See [29]) Let $\mathcal{P}>0$ and $\Omega \subseteq \mathbb{R}$ be an interval. Then $\Omega$ is said to be $\mathcal{P}$ convex if

$$
\left(\xi l_{1}^{\mathcal{P}}+(1-\xi) l_{2}^{\mathcal{P}}\right)^{1 / \mathcal{P}} \in \Omega
$$

for all $l_{1}, l_{2} \in \Omega$ and $\xi \in[0,1]$.

Definition 2.2 (See [29]) Let $\mathcal{P}>0$ and $\Omega \subseteq \mathbb{R}$ be a $\mathcal{P}$-convex interval. Then the realvalued function $\hbar: \Omega \rightarrow \mathbb{R}$ is said to be $\mathcal{P}$-convex if the inequality

$$
\hbar\left(\left[\xi l_{1}^{\mathcal{P}}+(1-\xi) l_{2}^{\mathcal{P}}\right]^{1 / \mathcal{P}}\right) \leq \xi \hbar\left(l_{1}\right)+(1-\xi) \hbar\left(l_{2}\right)
$$

holds for all $l_{1}, l_{2} \in \Omega$ and $\xi \in[0,1]$.

If $\mathcal{P}=1$, then we clearly see that the $\mathcal{P}$-convexity reduces to classical convexity.

Definition 2.3 (See [30]) Let $\Omega \subseteq \mathbb{R}$ be an interval. Then a real-valued function $\hbar: \Omega \rightarrow \mathbb{R}$ is said to be harmonically convex if the inequality

$$
\hbar\left(\frac{l_{1} l_{2}}{\xi l_{1}+(1-\xi) l_{2}}\right) \leq \xi \hbar\left(l_{2}\right)+(1-\xi) \hbar\left(l_{1}\right)
$$

holds for all $l_{1}, l_{2} \in \Omega$ and $\xi \in[0,1]$.

In [31], Toplu et al. defined the $n$-polynomial convexity as follows.

Definition 2.4 (See [31]) Let $n \in \mathbb{N}$. Then the non-negative function $\hbar: \Omega \rightarrow[0, \infty)$ is said to be a $n$-polynomial convex function if the inequality

$$
\hbar\left(\xi l_{1}+(1-\xi) l_{2}\right) \leq \frac{1}{n} \sum_{\theta=1}^{n}\left[1-(1-\xi)^{\theta}\right] \hbar\left(l_{1}\right)+\frac{1}{n} \sum_{\theta=1}^{n}\left[\left(1-\xi^{\theta}\right)\right] \hbar\left(l_{2}\right)
$$

holds for every $l_{1}, l_{2} \in \Omega$ and $\xi \in[0,1]$.

Note that every $n$-polynomial convex function is also a $\lambda$-convex function if $\lambda(\xi)=$ $\frac{1}{n} \sum_{\theta=1}^{n}\left[1-(1-\xi)^{\theta}\right]$.

Awan et al. [32] presented the idea of $n$-polynomial harmonically convex functions as follows.

Definition 2.5 (See [32]) Let $n \in \mathbb{N}$. Then a non-negative function $\hbar: \Omega \rightarrow[0, \infty)$ is said to be a $n$-polynomial harmonically convex function if the inequality

$$
\hbar\left(\frac{l_{1} l_{2}}{\xi l_{1}+(1-\xi) l_{2}}\right) \leq \frac{1}{n} \sum_{\theta=1}^{n}\left[1-(1-\xi)^{\theta}\right] \hbar\left(l_{2}\right)+\frac{1}{n} \sum_{\theta=1}^{n}\left[\left(1-\xi^{\theta}\right)\right] \hbar\left(l_{1}\right)
$$

holds for every $l_{1}, l_{2} \in \Omega$ and $\xi \in[0,1]$. 
If $n=2$, then from Definition 2.5 we clearly see that the $n$-polynomial harmonically convex function $\hbar$ satisfies the inequality

$$
\hbar\left(\frac{l_{1} l_{2}}{\xi l_{1}+(1-\xi) l_{2}}\right) \leq \frac{3 \xi-\xi^{2}}{2} \hbar\left(l_{2}\right)+\frac{2-\xi-\xi^{2}}{2} \hbar\left(l_{1}\right)
$$

for all $l_{1}, l_{2} \in \Omega$ and $\xi \in[0,1]$.

Now, we generalize the $n$-polynomial convex function to the $n$-polynomial $\mathcal{P}$-convex function.

Definition 2.6 Let $n \in \mathbb{N}, \mathcal{P}>0$ and $\Omega \subseteq \mathbb{R}$ be a $\mathcal{P}$-convex interval. Then the non-negative real-valued function $\hbar: \Omega \rightarrow[0, \infty)$ is said to be a $n$-polynomial $\mathcal{P}$-convex function if the inequality

$$
\hbar\left(\left[\xi l_{1}^{\mathcal{P}}+(1-\xi) l_{2}^{\mathcal{P}}\right]^{1 / \mathcal{P}}\right) \leq \frac{1}{n} \sum_{\theta=1}^{n}\left[1-(1-\xi)^{\theta}\right] \hbar\left(l_{1}\right)+\frac{1}{n} \sum_{\theta=1}^{n}\left[\left(1-\xi^{\theta}\right)\right] \hbar\left(l_{2}\right)
$$

holds for all $l_{1}, l_{2} \in \Omega, \xi \in[0,1]$.

Remark 2.7 Definition 2.6 leads to the conclusion that:

(i) If $\mathcal{P}=-1$, then Definition 2.6 becomes Definition 2.5 for $n$-polynomial harmonically convex function.

(ii) If $\mathcal{P}=1$, then Definition 2.6 reduces to Definition (2.4) for $n$-polynomial convex function.

Definition 2.8 (See [33]) Let $\eta, \rho>0$. Then the left and right sides generalized fractional integrals of the function $\hbar$ defined on $\left[l_{1}, l_{1}\right]$ are defined by

$$
\left({ }^{\rho} \mathcal{I}_{l_{1}^{+}}^{\eta} \hbar\right)(z)=\frac{\rho^{1-\eta}}{\Gamma(\eta)} \int_{l_{1}}^{z} \frac{\left(z^{\rho}-\xi^{\rho}\right)^{\eta-1} \hbar(\xi)}{\xi^{1-\rho}} d \xi \quad\left(z>l_{1}\right)
$$

and

$$
\left({ }^{\rho} \mathcal{I}_{l_{2}^{\eta}}^{\eta} \hbar\right)(z)=\frac{\rho^{1-\eta}}{\Gamma(\eta)} \int_{z}^{l_{2}} \frac{\left(\xi^{\rho}-z^{\rho}\right)^{\eta-1} \hbar(\xi)}{\xi^{1-\rho}} d \xi \quad\left(z<l_{2}\right),
$$

respectively.

Remark 2.9 Definition 2.8 leads to the conclusions that

(1) If $\rho=1$, then we get the Riemann-Liouville fractional integral operators [34]

$$
\left(\mathcal{I}_{l_{1}^{+}}^{\eta} \hbar\right)(z)=\frac{1}{\Gamma(\eta)} \int_{l_{1}}^{z}(z-\xi)^{\eta-1} \hbar(\xi) d \xi \quad\left(z>l_{1}\right)
$$

and

$$
\left(\mathcal{I}_{l_{2}^{-}}^{\eta} \hbar\right)(z)=\frac{1}{\Gamma(\eta)} \int_{z}^{l_{2}}(\xi-z)^{\eta-1} \hbar(\xi) d \xi \quad\left(z<l_{2}\right) .
$$


(2) It $\rho \rightarrow 0$, then we obtain the Hadamard fractional integral operators [34]

$$
\left(\mathcal{I}_{l_{1}^{+}}^{\eta} \hbar\right)(z)=\frac{1}{\Gamma(\eta)} \int_{l_{1}}^{z}\left(\log \frac{z}{\xi}\right)^{\eta-1} \frac{\hbar(\xi)}{\xi} d \xi \quad\left(z>l_{1}\right)
$$

and

$$
\left(\mathcal{I}_{l_{2}^{-}}^{\eta} \hbar\right)(z)=\frac{1}{\Gamma(\eta)} \int_{z}^{l_{2}}\left(\log \frac{\xi}{z}\right)^{\eta-1} \frac{\hbar(\xi)}{\xi} d \xi \quad\left(z<l_{2}\right) .
$$

Next, we also need to introduce the beta function $\mathbb{B}$ and Gaussian hypergeometric function ${ }_{2} \mathcal{F}_{1}$, defined by

$$
\mathbb{B}\left(z_{1}, z_{2}\right)=\frac{\Gamma\left(z_{1}\right) \Gamma\left(z_{2}\right)}{\Gamma\left(z_{1}+z_{2}\right)}=\int_{0}^{1} \xi^{z_{1}-1}(1-\xi)^{z_{2}-1} d \xi \quad\left(z_{1}, z_{2}>0\right)
$$

and

$$
\begin{aligned}
& { }_{2} \mathcal{F}_{1}\left(z_{1}, z_{2} ; z_{3}, z\right)=\frac{1}{\mathbb{B}\left(z_{2}, z_{3}-z_{2}\right)} \int_{0}^{1} \xi^{z_{2}-1}(1-\xi)^{z_{3}-z_{2}-1}(1-z \xi)^{-z_{1}} d \xi \\
& \left(z_{3}>z_{2}>0,|z|<1\right)
\end{aligned}
$$

respectively, where $\Gamma(z)=\int_{0}^{\infty} e^{-\xi} \xi^{z-1} d \xi$ is the Euler gamma function [35].

\section{Hermite-Hadamard type inequality for $\boldsymbol{n}$-polynomial $\mathcal{P}$-convex function}

In this section, we establish the Hermite-Hadamard type inequality for $n$-polynomial $\mathcal{P}$ convex functions via generalized fractional integrals.

Theorem 3.1 Let $n \in \mathbb{N}, \mathcal{P}>0, \eta>0, \Omega \subset(0, \infty)$ be a $\mathcal{P}$-convex interval, $l_{1}, l_{2} \in \Omega$ with $l_{1}<l_{2}$ and $\hbar: \Omega \rightarrow \mathbb{R}$ be an n-polynomial $\mathcal{P}$-convex function such that $\hbar \in L_{1}\left(\left[l_{1}, l_{2}\right]\right)$. Then we have

$$
\begin{aligned}
\frac{2^{n} n}{\left(2^{n}(n-1)+1\right)} \hbar\left(\sqrt[\mathcal{P}]{\frac{l_{1}^{\mathcal{P}}+l_{2}^{\mathcal{P}}}{2}}\right) & \leq \frac{\mathcal{P}^{\eta} \Gamma(\eta+1)}{2\left(l_{2}^{\mathcal{P}}-l_{1}^{\mathcal{P}}\right)^{\eta}}\left[{ }^{\mathcal{P}} \mathcal{I}_{l_{1}^{+}}^{\eta} \hbar\left(l_{2}\right)+{ }^{\mathcal{P}} \mathcal{I}_{l_{2}}^{\eta} \hbar\left(l_{1}\right)\right] \\
& \leq \frac{\hbar\left(l_{1}\right)+\hbar\left(l_{2}\right)}{2 n} \sum_{\theta=1}^{n}\left[\frac{\eta+2 \theta}{\eta(\eta+\theta)}-\mathbb{B}(\theta+1, \eta)\right] .
\end{aligned}
$$

Proof Let $x, y \in \Omega$ and $\xi=\frac{1}{2}$ in (2.3). Then it follows from the $n$-polynomial $\mathcal{P}$-convexity of the function $\hbar$ on $\Omega$ that

$$
\hbar\left(\mathcal{P} \sqrt{\frac{x^{\mathcal{P}}+y^{\mathcal{P}}}{2}}\right) \leq \frac{1}{n} \sum_{\theta=1}^{n}\left(1-\left(\frac{1}{2}\right)^{\theta}\right)[\hbar(x)+\hbar(y)] .
$$

Putting $x^{\mathcal{P}}=\xi l_{1}^{\mathcal{P}}+(1-\xi) l_{2}^{\mathcal{P}}$ and $y^{\mathcal{P}}=(1-\xi) l_{1}^{\mathcal{P}}+\xi l_{2}^{\mathcal{P}}$ leads to

$$
\begin{aligned}
& \hbar\left(\sqrt[\mathcal{P}]{\frac{l_{1}^{\mathcal{P}}+l_{2}^{\mathcal{P}}}{2}}\right) \\
& \quad \leq \frac{1}{n} \sum_{\theta=1}^{n}\left(1-\left(\frac{1}{2}\right)^{\theta}\right)\left[\hbar\left(\mathcal{P}^{\mathcal{P}} \sqrt{\xi l_{1}^{\mathcal{P}}+(1-\xi) l_{2}^{\mathcal{P}}}\right)+\hbar\left(\mathcal{P} \sqrt{(1-\xi) l_{1}^{\mathcal{P}}+\xi l_{2}^{\mathcal{P}}}\right)\right] .
\end{aligned}
$$


Multiplying both sides of (3.3) by $\xi^{\eta-1}$ and integrating the obtained inequality with respect to $\xi$ over $(0,1)$ give

$$
\begin{aligned}
& \frac{2^{n+1} n}{\eta\left(2^{n}(n-1)+1\right)} \hbar\left(\sqrt{\frac{l_{1}^{\mathcal{P}}+l_{2}^{\mathcal{P}}}{2}}\right) \\
& \leq \int_{0}^{1} \xi^{\eta-1} \hbar\left(\mathcal{P} \sqrt{\xi l_{1}^{\mathcal{P}}+(1-\xi) l_{2}^{\mathcal{P}}}\right) d \xi+\int_{0}^{1} \xi^{\eta-1} \hbar\left(\mathcal{P} \sqrt{(1-\xi) l_{1}^{\mathcal{P}}+\xi l_{2}^{\mathcal{P}}}\right) d \xi \\
& =\frac{\mathcal{P}}{l_{2}^{\mathcal{P}}-l_{1}^{\mathcal{P}}}\left[\int_{l_{1}}^{l_{2}}\left(\frac{l_{2}^{\mathcal{P}}-z^{\mathcal{P}}}{l_{2}^{\mathcal{P}}-l_{1}^{\mathcal{P}}}\right)^{\eta-1} \frac{\hbar(z)}{z^{1-\mathcal{P}}} d z+\int_{l_{1}}^{l_{2}}\left(\frac{z^{\mathcal{P}}-l_{1}^{\mathcal{P}}}{l_{2}^{\mathcal{P}}-l_{1}^{\mathcal{P}}}\right)^{\eta-1} \frac{\hbar(z)}{z^{1-\mathcal{P}}} d z\right] \\
& =\frac{\mathcal{P}^{\eta} \Gamma(\eta)}{\left(l_{2}^{\mathcal{P}}-l_{1}^{\mathcal{P}}\right)^{\eta}}\left[{ }^{\mathcal{P}} \mathcal{I}_{l_{1}^{\eta}}^{\eta} \hbar\left(l_{2}\right)+{ }^{\mathcal{P}} \mathcal{I}_{l_{2}^{\eta}}^{\eta} \hbar\left(l_{1}\right)\right],
\end{aligned}
$$

that is,

$$
\frac{2^{n} n}{\left(2^{n}(n-1)+1\right)} \hbar\left(\mathcal{P} \sqrt{\frac{l_{1}^{\mathcal{P}}+l_{2}^{\mathcal{P}}}{2}}\right) \leq \frac{\mathcal{P}^{\eta} \Gamma(\eta+1)}{2\left(l_{2}^{\mathcal{P}}-l_{1}^{\mathcal{P}}\right)^{\eta}}\left[{ }^{\mathcal{P}} \mathcal{I}_{l_{1}^{+}}^{\eta} \hbar\left(l_{2}\right)+{ }^{\mathcal{P}} \mathcal{I}_{l_{2}^{\eta}}^{\eta} \hbar\left(l_{1}\right)\right]
$$

which completes the proof of the first inequality. For the proof of the second inequality of (3.1), we use the $n$-polynomial $\mathcal{P}$-convexity of the function $\hbar$ again to get

$$
\hbar\left(\mathcal{P} \sqrt{\xi l_{1}^{\mathcal{P}}+(1-\xi) l_{2}^{\mathcal{P}}}\right) \leq \frac{1}{n} \sum_{\theta=1}^{n}\left[1-(1-\xi)^{\theta}\right] \hbar\left(l_{1}\right)+\frac{1}{n} \sum_{\theta=1}^{n}\left[1-\xi^{\theta}\right] \hbar\left(l_{2}\right)
$$

and

$$
\hbar\left(\mathcal{P} \sqrt{(1-\xi) l_{1}^{\mathcal{P}}+\xi l_{2}^{\mathcal{P}}}\right) \leq \frac{1}{n} \sum_{\theta=1}^{n}\left[1-\xi^{\theta}\right] \hbar\left(l_{1}\right)+\frac{1}{n} \sum_{\theta=1}^{n}\left[1-(1-\xi)^{\theta}\right] \hbar\left(l_{2}\right) .
$$

By adding the above two inequalities, we have

$$
\begin{aligned}
\hbar( & \left(\mathcal{P} \sqrt{(1-\xi) l_{1}^{\mathcal{P}}+\xi l_{2}^{\mathcal{P}}}\right)+\hbar\left(\mathcal{P} \sqrt{\xi l_{1}^{\mathcal{P}}+(1-\xi) l_{2}^{\mathcal{P}}}\right) \\
& \leq\left[\frac{1}{n} \sum_{\theta=1}^{n}\left[1-(1-\xi)^{\theta}\right]+\frac{1}{n} \sum_{\theta=1}^{n}\left[1-\xi^{\theta}\right]\right]\left[\hbar\left(l_{1}\right)+\hbar\left(l_{2}\right)\right] .
\end{aligned}
$$

Multiplying both sides of the (3.6) by $\xi^{\eta-1}$ and integrating the obtained inequality for $\xi$ over $(0,1)$, we obtain

$$
\frac{\mathcal{P}^{\eta} \Gamma(\eta+1)}{2\left(l_{2}^{\mathcal{P}}-l_{1}^{\mathcal{P}}\right)^{\eta}}\left[{ }^{\mathcal{P}} \mathcal{I}_{l_{1}^{+}}^{\eta} \hbar\left(l_{2}\right)+{ }^{\mathcal{P}} \mathcal{I}_{l_{2}^{\eta}}^{\eta} \hbar\left(l_{1}\right)\right] \leq \frac{1}{n} \sum_{\theta=1}^{n}\left[\frac{\eta+2 \theta}{\eta(\eta+\theta)}-\mathbb{B}(\theta+1, \eta)\right] \frac{\hbar\left(l_{1}\right)+\hbar\left(l_{2}\right)}{2} .
$$

Remark 3.2 Theorem 3.1 leads to the conclusions that:

(1) If $\eta=\mathcal{P}=1$, then we obtain Theorem 4 of [31].

(2) If $\eta=\mathcal{P}=-1$, then we get Theorem 2.3 of [32]. 


\section{Hermite-Hadamard type inequalities for differentiable function}

In what follows, we denote by $L_{1}\left(\left[l_{1}, l_{2}\right]\right)$ the space of (Lebesgue) integrable functions on the interval $\left[l_{1}, l_{2}\right]$, we first give Lemma 4.1 [36], which will be used for generating refinements of Hermite-Hadamard type inequality.

Lemma 4.1 (See [36]) Let $\mathcal{P}, \eta>0, \hbar: \Omega^{\circ} \subset(0, \infty) \rightarrow \mathbb{R}$ be a differentiable function on $\Omega^{\circ}$ $\left(\Omega^{\circ}\right.$ is the interior of $\Omega$ ) and $l_{1}, l_{2} \in \Omega^{\circ}$ with $l_{1}<l_{2}$ such that $\hbar^{\prime} \in L_{1}\left(\left[l_{1}, l_{2}\right]\right)$. Then we have the inequality

$$
\begin{aligned}
& \frac{\hbar\left(l_{1}\right)+\hbar\left(l_{2}\right)}{2}-\frac{\mathcal{P}^{\eta} \Gamma(\eta+1)}{2\left(l_{2}^{\mathcal{P}}-l_{1}^{\mathcal{P}}\right)^{\eta}}\left[{ }^{\mathcal{P}} \mathcal{I}_{l_{1}^{+}}^{\eta} \hbar\left(l_{2}\right)+{ }^{\mathcal{P}} \mathcal{I}_{l_{2}^{\eta}}^{\eta} \hbar\left(l_{1}\right)\right] \\
& \quad=\frac{l_{2}^{\mathcal{P}}-l_{1}^{\mathcal{P}}}{2 \mathcal{P}} \int_{0}^{1}\left[(1-\xi)^{\eta}-\xi^{\eta}\right]\left(\xi l_{1}^{\mathcal{P}}+(1-\xi) l_{2}^{\mathcal{P}}\right)^{\frac{1-\mathcal{P}}{\mathcal{P}}} \hbar^{\prime}\left(\mathcal{P} \sqrt{\xi l_{1}^{\mathcal{P}}+(1-\xi) l_{2}^{\mathcal{P}}}\right) d \xi .
\end{aligned}
$$

Theorem 4.2 Let $n \in \mathbb{N}, \mathcal{P}, \eta>0, \beta>1, \hbar: \Omega^{\circ} \subset(0, \infty) \rightarrow \mathbb{R}$ be a differentiable function on $\Omega^{\circ}$ and $l_{1}, l_{2} \in \Omega^{\circ}$ with $l_{1}<l_{2}$ such that $\hbar^{\prime} \in L_{1}\left(\left[l_{1}, l_{2}\right]\right)$ and $\left|\hbar^{\prime}\right|^{\beta}$ is an n-polynomial $\mathcal{P}$-convex function on $\Omega$. Then one has

$$
\begin{aligned}
& \left|\frac{\hbar\left(l_{1}\right)+\hbar\left(l_{2}\right)}{2}-\frac{\mathcal{P}^{\eta} \Gamma(\eta+1)}{2\left(l_{2}^{\mathcal{P}}-l_{1}^{\mathcal{P}}\right)^{\eta}}\left[{ }^{\mathcal{P}} \mathcal{I}_{l_{1}^{+}}^{\eta} \hbar\left(l_{2}\right)+{ }^{\mathcal{P}} \mathcal{I}_{l_{2}}^{\eta} \hbar\left(l_{1}\right)\right]\right| \\
& \leq \frac{l_{2}^{\mathcal{P}}-l_{1}^{\mathcal{P}}}{2 \mathcal{P}}\left(\Psi_{1}\left(l_{1}, l_{2} ; \mathcal{P}\right)\right)^{1-1 / \beta} \\
& \quad \times\left(\frac{\left|\hbar\left(l_{1}\right)\right|^{\beta}}{n} \sum_{\theta=1}^{n} \Psi_{2}\left(l_{1}, l_{2} ; \mathcal{P}\right)+\frac{\left|\hbar\left(l_{2}\right)\right|^{\beta}}{n} \sum_{\theta=1}^{n} \Psi_{3}\left(l_{1}, l_{2} ; \mathcal{P}\right)\right)^{1 / \beta},
\end{aligned}
$$

where

$$
\begin{aligned}
& \Psi_{1}\left(l_{1}, l_{2} ; \mathcal{P}\right)=\frac{1}{l_{2}^{(\mathcal{P}-1)}(\eta+1)}\left\{\begin{array}{l}
{\left[{ }_{2} \mathcal{F}_{1}\left((1-1 / \mathcal{P}), 1, \eta+2,1-\left(l_{1} / l_{2}\right)^{\mathcal{P}}\right)\right.} \\
\left.-{ }_{2} \mathcal{F}_{1}\left((1-1 / \mathcal{P}), \eta+1, \eta+2,1-\left(l_{1} / l_{2}\right)^{\mathcal{P}}\right)\right]
\end{array}\right. \\
& \Psi_{2}\left(l_{1}, l_{2} ; \mathcal{P}\right)=\frac{1}{l_{2}^{(\mathcal{P}-1)}}\left\{\begin{array}{c}
\frac{1}{\eta+1}\left[{ }_{2} \mathcal{F}_{1}\left((1-1 / \mathcal{P}), 1, \eta+2,1-\left(l_{1} / l_{2}\right)^{\mathcal{P}}\right)\right. \\
\left.\quad-{ }_{2} \mathcal{F}_{1}\left((1-1 / \mathcal{P}), \eta+1, \eta+2,1-\left(l_{1} / l_{2}\right)^{\mathcal{P}}\right)\right] \\
+\mathbb{B}(\eta+1, \theta+1)\left[\mathcal{F}_{1}\left((1-1 / \mathcal{P}), \eta+1, \theta+\eta+2,1-\left(l_{1} / l_{2}\right)^{\mathcal{P}}\right)\right] \\
-\frac{1}{\eta+\theta+2}\left[{ }_{2} \mathcal{F}_{1}\left((1-1 / \mathcal{P}), 1, \theta+\eta+2,1-\left(l_{1} / l_{2}\right)^{\mathcal{P}}\right)\right]
\end{array}\right.
\end{aligned}
$$

and

$$
\Psi_{3}\left(l_{1}, l_{2} ; \mathcal{P}\right)=\frac{1}{l_{2}^{(\mathcal{P}-1)}}\left\{\begin{aligned}
\frac{1}{\eta+1}\left[{ }_{2}\right. & \mathcal{F}_{1}\left((1-1 / \mathcal{P}), 1, \eta+2,1-\left(l_{1} / l_{2}\right)^{\mathcal{P}}\right) \\
& \left.-{ }_{2} \mathcal{F}_{1}\left((1-1 / \mathcal{P}), \eta+2, \eta+1,1-\left(l_{1} / l_{2}\right)^{\mathcal{P}}\right)\right] \\
& -\mathbb{B}(\eta+1, \theta+1)\left[{ }_{2} \mathcal{F}_{1}\left((1-1 / \mathcal{P}), \theta+1, \theta+\eta+2,1-\left(l_{1} / l_{2}\right)^{\mathcal{P}}\right)\right] \\
& +\frac{1}{\eta+\theta+2}\left[{ }_{2} \mathcal{F}_{1}\left((1-1 / \mathcal{P}), \eta+\theta+1, \theta+\eta+2,1-\left(l_{1} / l_{2}\right)^{\mathcal{P}}\right)\right] .
\end{aligned}\right.
$$


Proof It follows from Lemma 4.1 and the Hölder inequality that

$$
\begin{aligned}
& \left|\frac{\hbar\left(l_{1}\right)+\hbar\left(l_{2}\right)}{2}-\frac{\mathcal{P}^{\eta} \Gamma(\eta+1)}{2\left(l_{2}^{\mathcal{P}}-l_{1}^{\mathcal{P}}\right)^{\eta}}\left[{ }^{\mathcal{P}} \mathcal{I}_{l_{1}^{+}}^{\eta} \hbar\left(l_{2}\right)+{ }^{\mathcal{P}} \mathcal{I}_{l_{2}}^{\eta} \hbar\left(l_{1}\right)\right]\right| \\
& \leq \frac{l_{2}^{\mathcal{P}}-l_{1}^{\mathcal{P}}}{2 \mathcal{P}} \int_{0}^{1}\left[(1-\xi)^{\eta}-\xi^{\eta}\right]\left(\xi l_{1}^{\mathcal{P}}+(1-\xi) l_{2}^{\mathcal{P}}\right)^{\frac{1-\mathcal{P}}{\mathcal{P}}}\left|\hbar^{\prime}\left(\sqrt[\mathcal{P}]{\xi l_{1}^{\mathcal{P}}+(1-\xi) l_{2}^{\mathcal{P}}}\right)\right| d \xi \\
& \leq \frac{l_{2}^{\mathcal{P}}-l_{1}^{\mathcal{P}}}{2 \mathcal{P}}\left(\int_{0}^{1}\left[(1-\xi)^{\eta}-\xi^{\eta}\right]\left(\xi l_{1}^{\mathcal{P}}+(1-\xi) l_{2}^{\mathcal{P}}\right)^{\frac{1-\mathcal{P}}{\mathcal{P}}} d \xi\right)^{1-1 / \beta} \\
& \quad \times\left(\int_{0}^{1}\left[(1-\xi)^{\eta}-\xi^{\eta}\right]\left(\xi l_{1}^{\mathcal{P}}+(1-\xi) l_{2}^{\mathcal{P}}\right)^{\frac{1-\mathcal{P}}{\mathcal{P}}}\left|\hbar^{\prime}\left(\sqrt{\mathcal{P}} \sqrt{\xi l_{1}^{\mathcal{P}}+(1-\xi) l_{2}^{\mathcal{P}}}\right)\right|^{\beta} d \xi\right)^{1 / \beta} .
\end{aligned}
$$

From the $n$-polynomial $\mathcal{P}$-convexity of the function $\left|\hbar^{\prime}\right|^{\beta}$ on $\Omega$, we get

$$
\begin{aligned}
\int_{0}^{1} & {\left[(1-\xi)^{\eta}-\xi^{\eta}\right]\left(\xi l_{1}^{\mathcal{P}}+(1-\xi) l_{2}^{\mathcal{P}}\right)^{\frac{1-\mathcal{P}}{\mathcal{P}}}\left|\hbar^{\prime}\left(\mathcal{P} \sqrt{\xi l_{1}^{\mathcal{P}}+(1-\xi) l_{2}^{\mathcal{P}}}\right)\right|^{\beta} d \xi } \\
\leq & \int_{0}^{1}\left[(1-\xi)^{\eta}-\xi^{\eta}\right]\left(\xi l_{1}^{\mathcal{P}}+(1-\xi) l_{2}^{\mathcal{P}}\right)^{\frac{1-\mathcal{P}}{\mathcal{P}}} \\
& \times\left[\frac{1}{n} \sum_{\theta=1}^{n}\left[1-(1-\xi)^{\theta}\right]\left|\hbar\left(l_{1}\right)\right|^{\beta}+\frac{1}{n} \sum_{\theta=1}^{n}\left[1-\xi^{\theta}\right]\left|\hbar\left(l_{2}\right)\right|^{\beta}\right] d \xi \\
\leq & \frac{\left|\hbar\left(l_{1}\right)\right|^{\beta}}{n} \sum_{\theta=1}^{n}\left[\int_{0}^{1}\left(\xi l_{1}^{\mathcal{P}}+(1-\xi) l_{2}^{\mathcal{P}}\right)^{\frac{1-\mathcal{P}}{\mathcal{P}}}\left[(1-\xi)^{\eta}-(1-\xi)^{\eta+\theta}-\xi^{\eta}+\xi^{\eta}(1-\xi)^{\theta}\right]\right] d \xi \\
& +\frac{\left|\hbar\left(l_{2}\right)\right|^{\beta}}{n} \sum_{\theta=1}^{n}\left[\int_{0}^{1}\left(\xi l_{1}^{\mathcal{P}}+(1-\xi) l_{2}^{\mathcal{P}}\right)^{\frac{1-\mathcal{P}}{\mathcal{P}}}\left[(1-\xi)^{\eta}-\xi^{\theta}(1-\xi)^{\eta}-\xi^{\eta}+\xi^{\eta+\theta}\right]\right] d \xi \\
= & \frac{\left|\hbar\left(l_{1}\right)\right|^{\beta}}{n} \sum_{\theta=1}^{n} \Psi_{2}\left(l_{1}, l_{2} ; \mathcal{P}\right)+\frac{\left|\hbar\left(l_{2}\right)\right|^{\beta}}{n} \sum_{\theta=1}^{n} \Psi_{3}\left(l_{1}, l_{2} ; \mathcal{P}\right) .
\end{aligned}
$$

Inequalities (4.3) and (4.4) lead to

$$
\begin{aligned}
& \left|\frac{\hbar\left(l_{1}\right)+\hbar\left(l_{2}\right)}{2}-\frac{\mathcal{P}^{\eta} \Gamma(\eta+1)}{2\left(l_{2}^{\mathcal{P}}-l_{1}^{\mathcal{P}}\right)^{\eta}}\left[{ }^{\mathcal{P}} \mathcal{I}_{l_{1}^{+}}^{\eta} \hbar\left(l_{2}\right)+{ }^{\mathcal{P}} \mathcal{I}_{l_{2}^{-}}^{\eta} \hbar\left(l_{1}\right)\right]\right| \\
& \leq \frac{l_{2}^{\mathcal{P}}-l_{1}^{\mathcal{P}}}{2 \mathcal{P}}\left(\Psi_{1}\left(l_{1}, l_{2} ; \mathcal{P}\right)\right)^{1-1 / \beta} \\
& \quad \times\left(\frac{\left|\hbar\left(l_{1}\right)\right|^{\beta}}{n} \sum_{\theta=1}^{n} \Psi_{2}\left(l_{1}, l_{2} ; \mathcal{P}\right)+\frac{\left|\hbar\left(l_{2}\right)\right|^{\beta}}{n} \sum_{\theta=1}^{n} \Psi_{3}\left(l_{1}, l_{2} ; \mathcal{P}\right)\right)^{1 / \beta}
\end{aligned}
$$

where we have used the facts that

$$
\begin{aligned}
\Psi_{1}\left(l_{1}, l_{2} ; \mathcal{P}\right) & :=\int_{0}^{1}\left[(1-\xi)^{\eta}-\xi^{\eta}\right]\left(\xi l_{1}^{\mathcal{P}}+(1-\xi) l_{2}^{\mathcal{P}}\right)^{\frac{1-\mathcal{P}}{\mathcal{P}}} d \xi \\
& =\frac{1}{l_{2}^{(\mathcal{P}-1)}(\eta+1)}\left\{\begin{array}{c}
{\left[\mathcal{F}_{1}\left((1-1 / \mathcal{P}), 1, \eta+2,1-\left(l_{1} / l_{2}\right)^{\mathcal{P}}\right)\right.} \\
\left.-{ }_{2} \mathcal{F}_{1}\left((1-1 / \mathcal{P}), \eta+1, \eta+2,1-\left(l_{1} / l_{2}\right)^{\mathcal{P}}\right)\right]
\end{array}\right.
\end{aligned}
$$




$$
\begin{gathered}
\Psi_{2}\left(l_{1}, l_{2} ; \mathcal{P}\right):=\int_{0}^{1}\left(\xi l_{1}^{\mathcal{P}}+(1-\xi) l_{2}^{\mathcal{P}}\right)^{\frac{1-\mathcal{P}}{\mathcal{P}}}\left[(1-\xi)^{\eta}-(1-\xi)^{\eta+\theta}-\xi^{\eta}+\xi^{\eta}(1-\xi)^{\theta}\right] d \xi \\
=\frac{1}{l_{2}^{(\mathcal{P}-1)}}\left\{\begin{array}{c}
\frac{1}{\eta+1}\left[{ }_{2} \mathcal{F}_{1}\left((1-1 / \mathcal{P}), 1, \eta+2,1-\left(l_{1} / l_{2}\right)^{\mathcal{P}}\right)\right. \\
\left.-{ }_{2} \mathcal{F}_{1}\left((1-1 / \mathcal{P}), \eta+1, \eta+2,1-\left(l_{1} / l_{2}\right)^{\mathcal{P}}\right)\right] \\
+\mathbb{B}(\eta+1, \theta+1) \\
\times\left[{ }_{2} \mathcal{F}_{1}\left((1-1 / \mathcal{P}), \eta+1, \theta+\eta+2,1-\left(l_{1} / l_{2}\right)^{\mathcal{P}}\right)\right] \\
-\frac{1}{\eta+\theta+2}\left[{ }_{2} \mathcal{F}_{1}\left((1-1 / \mathcal{P}), 1, \theta+\eta+2,1-\left(l_{1} / l_{2}\right)^{\mathcal{P}}\right)\right]
\end{array}\right.
\end{gathered}
$$

and

$$
\begin{aligned}
\Psi_{3}\left(l_{1}, l_{2} ; \mathcal{P}\right):=\int_{0}^{1}\left(\xi l_{1}^{\mathcal{P}}+(1-\xi) l_{2}^{\mathcal{P}}\right)^{\frac{1-\mathcal{P}}{\mathcal{P}}}\left[(1-\xi)^{\eta}-\xi^{\theta}(1-\xi)^{\eta}-\xi^{\eta}+\xi^{\eta+\theta}\right] d \xi \\
=\frac{1}{l_{2}^{(\mathcal{P}-1)}}\left\{\begin{array}{c}
\frac{1}{\eta+1}\left[{ }_{2} \mathcal{F}_{1}\left((1-1 / \mathcal{P}), 1, \eta+2,1-\left(l_{1} / l_{2}\right)^{\mathcal{P}}\right)\right. \\
\left.-{ }_{2} \mathcal{F}_{1}\left((1-1 / \mathcal{P}), \eta+2, \eta+1,1-\left(l_{1} / l_{2}\right)^{\mathcal{P}}\right)\right] \\
-\mathbb{B}(\eta+1, \theta+1) \\
\times\left[{ }_{2} \mathcal{F}_{1}\left((1-1 / \mathcal{P}), \theta+1, \theta+\eta+2,1-\left(l_{1} / l_{2}\right)^{\mathcal{P}}\right)\right] \\
+\frac{1}{\eta+\theta+2}\left[2 \mathcal{F}_{1}\left((1-1 / \mathcal{P}), \eta+\theta+1, \theta+\eta+2,1-\left(l_{1} / l_{2}\right)^{\mathcal{P}}\right)\right]
\end{array}\right.
\end{aligned}
$$

Combining (4.5)-(4.8), we get the desired inequality (4.2).

Our next result is better than all the previously known results in the literature, which can be obtained by the improved power-mean inequality via $n$-polynomial $\mathcal{P}$-convex function.

Theorem 4.3 Let $n \in \mathbb{N}, \mathcal{P}, \eta>0, \beta \geq 1, \hbar: \Omega^{\circ} \subset(0, \infty) \rightarrow \mathbb{R}$ be a differentiable function on $\Omega^{\circ}$ and $l_{1}, l_{2} \in \Omega^{\circ}$ with $l_{1}<l_{2}$ such that $\hbar^{\prime} \in L_{1}\left(\left[l_{1}, l_{2}\right]\right)$ and $\left|\hbar^{\prime}\right|^{\beta}$ is a n-polynomial $\mathcal{P}$ convex function on $\Omega$. Then

$$
\begin{aligned}
& \left.\mid \frac{\hbar\left(l_{1}\right)+\hbar\left(l_{2}\right)}{2}-\frac{\mathcal{P}^{\eta} \Gamma(\eta+1)}{2\left(l_{2}^{\mathcal{P}}-l_{1}^{\mathcal{P}}\right)^{\eta}}{ }^{\mathcal{P}} \mathcal{I}_{l_{1}^{\eta}}^{\eta} \hbar\left(l_{2}\right)+{ }^{\mathcal{P}} \mathcal{I}_{l_{2}^{\eta}}^{\eta} \hbar\left(l_{1}\right)\right] \mid \\
& \leq \frac{l_{2}^{\mathcal{P}}-l_{1}^{\mathcal{P}}}{2 \mathcal{P}} \\
& \quad \times\left[\left(\Psi_{1}^{*}\left(l_{1}, l_{2} ; \mathcal{P}\right)\right)^{1-1 / \alpha}\left(\frac{\left|\hbar\left(l_{1}\right)\right|^{\beta}}{n} \sum_{\theta=1}^{n} \Psi_{2}^{*}\left(l_{1}, l_{2} ; \mathcal{P}\right)+\frac{\left|\hbar\left(l_{2}\right)\right|^{\beta}}{n} \sum_{\theta=1}^{n} \Psi_{3}^{*}\left(l_{1}, l_{2} ; \mathcal{P}\right)\right)^{1 / \beta}\right. \\
& \left.\quad+\left(\Psi_{4}^{*}\left(l_{1}, l_{2} ; \mathcal{P}\right)\right)^{1-1 / \alpha}\left(\frac{\left|\hbar\left(l_{1}\right)\right|^{\beta}}{n} \sum_{\theta=1}^{n} \Psi_{5}^{*}\left(l_{1}, l_{2} ; \mathcal{P}\right)+\frac{\left|\hbar\left(l_{2}\right)\right|^{\beta}}{n} \sum_{\theta=1}^{n} \Psi_{6}^{*}\left(l_{1}, l_{2} ; \mathcal{P}\right)\right)^{1 / \beta}\right],
\end{aligned}
$$

where

$$
\Psi_{1}^{*}\left(l_{1}, l_{2} ; \mathcal{P}\right)=\frac{1}{l_{2}^{(\mathcal{P}-1)}}\left\{\begin{array}{c}
\frac{1}{\eta+2} 2 \mathcal{F}_{1}\left((1-1 / \mathcal{P}), 1, \eta+3,1-\left(l_{1} / l_{2}\right)^{\mathcal{P}}\right) \\
-\frac{1}{(\eta+2)(\eta+3)}{ }^{2} \mathcal{F}_{1}\left((1-1 / \mathcal{P}), \eta+1, \eta+3,1-\left(l_{1} / l_{2}\right)^{\mathcal{P}}\right)
\end{array}\right.
$$




$$
\begin{aligned}
& \Psi_{2}^{*}\left(l_{1}, l_{2} ; \mathcal{P}\right)=\frac{1}{l_{2}^{(\mathcal{P}-1)}}\left\{\begin{array}{l}
\frac{1}{\eta+1}\left[{ }_{2} \mathcal{F}_{1}\left((1-1 / \mathcal{P}), 1, \eta+3,1-\left(l_{1} / l_{2}\right)^{\mathcal{P}}\right)\right. \\
- \\
\left.-\frac{1}{\eta+2} 2 \mathcal{F}_{1}\left((1-1 / \mathcal{P}), \eta+1, \eta+3,1-\left(l_{1} / l_{2}\right)^{\mathcal{P}}\right)\right] \\
+\mathbb{B}(\eta+1, \theta+1)\left[2 \mathcal{F}_{1}\left((1-1 / \mathcal{P}), \eta+1, \theta+\eta+3,1-\left(l_{1} / l_{2}\right)^{\mathcal{P}}\right)\right] \\
-\frac{1}{\eta+\theta+1}\left[\mathcal{F}_{1}\left((1-1 / \mathcal{P}), 1, \theta+\eta+3,1-\left(l_{1} / l_{2}\right)^{\mathcal{P}}\right)\right],
\end{array}\right. \\
& \Psi_{3}^{*}\left(l_{1}, l_{2} ; \mathcal{P}\right)=\frac{1}{l_{2}^{(\mathcal{P}-1)}}\left\{\begin{array}{l}
\frac{1}{\eta+1}\left[{ }_{2} \mathcal{F}_{1}\left((1-1 / \mathcal{P}), 1, \eta+3,1-\left(l_{1} / l_{2}\right)^{\mathcal{P}}\right)\right. \\
\left.-\frac{1}{\eta+2} 2 \mathcal{F}_{1}\left((1-1 / \mathcal{P}), \eta+1, \eta+3,1-\left(l_{1} / l_{2}\right)^{\mathcal{P}}\right)\right] \\
-\mathbb{B}(\eta+2, \theta+1)\left[2 \mathcal{F}_{1}\left((1-1 / \mathcal{P}), \eta+1, \theta+\eta+3,1-\left(l_{1} / l_{2}\right)^{\mathcal{P}}\right)\right] \\
+\frac{1}{\eta+\theta+1}\left[2 \mathcal{F}_{1}\left((1-1 / \mathcal{P}), \theta+\eta+1, \theta+\eta+3,1-\left(l_{1} / l_{2}\right)^{\mathcal{P}}\right)\right],
\end{array}\right. \\
& \Psi_{4}^{*}\left(l_{1}, l_{2} ; \mathcal{P}\right)=\frac{1}{l_{2}^{(\mathcal{P}-1)}}\left\{\begin{array}{l}
\frac{1}{(\eta+1)(\eta+2)} 2 \mathcal{F}_{1}\left((1-1 / \mathcal{P}), 2, \eta+3,1-\left(l_{1} / l_{2}\right)^{\mathcal{P}}\right) \\
-\frac{1}{(\eta+2)}{ }^{2} \mathcal{F}_{1}\left((1-1 / \mathcal{P}), \eta+2, \eta+3,1-\left(l_{1} / l_{2}\right)^{\mathcal{P}}\right),
\end{array}\right. \\
& \Psi_{5}^{*}\left(l_{1}, l_{2} ; \mathcal{P}\right)=\frac{1}{l_{2}^{(\mathcal{P}-1)}}\left\{\begin{array}{l}
\frac{1}{\eta+1} 2 \mathcal{F}_{1}\left((1-1 / \mathcal{P}), 1, \eta+2,1-\left(l_{1} / l_{2}\right)^{\mathcal{P}}\right) \\
\left.-\frac{1}{\eta+2} 2 \mathcal{F}_{1}\left((1-1 / \mathcal{P}), \eta+2, \eta+3,1-\left(l_{1} / l_{2}\right)^{\mathcal{P}}\right)\right] \\
+\mathbb{B}(\eta+2, \theta+1)\left[{ }_{2} \mathcal{F}_{1}\left((1-1 / \mathcal{P}), \eta+2, \theta+\eta+3,1-\left(l_{1} / l_{2}\right)^{\mathcal{P}}\right)\right] \\
-\frac{1}{\eta+\theta+1}\left[2 \mathcal{F}_{1}\left((1-1 / \mathcal{P}), 2, \theta+\eta+3,1-\left(l_{1} / l_{2}\right)^{\mathcal{P}}\right)\right],
\end{array}\right.
\end{aligned}
$$

and

$$
\Psi_{6}^{*}\left(l_{1}, l_{2} ; \mathcal{P}\right)=\frac{1}{l_{2}^{(\mathcal{P}-1)}}\left\{\begin{array}{l}
\frac{1}{\eta+1} 2 \mathcal{F}_{1}\left((1-1 / \mathcal{P}), 2, \eta+3,1-\left(l_{1} / l_{2}\right)^{\mathcal{P}}\right) \\
\left.-\frac{1}{\eta+2} 2 \mathcal{F}_{1}\left((1-1 / \mathcal{P}), \eta+2, \eta+3,1-\left(l_{1} / l_{2}\right)^{\mathcal{P}}\right)\right] \\
-\mathbb{B}(\eta+1, \theta+2)\left[2 \mathcal{F}_{1}\left((1-1 / \mathcal{P}), \eta+2, \theta+\eta+3,1-\left(l_{1} / l_{2}\right)^{\mathcal{P}}\right)\right] \\
-\frac{1}{\eta+\theta+2}\left[{ }_{2} \mathcal{F}_{1}\left((1-1 / \mathcal{P}), \theta+\eta+2, \theta+\eta+3,1-\left(l_{1} / l_{2}\right)^{\mathcal{P}}\right)\right] .
\end{array}\right.
$$

Proof It follows from Lemma 4.1 and the improved power-mean integral inequality that

$$
\begin{aligned}
& \left|\frac{\hbar\left(l_{1}\right)+\hbar\left(l_{2}\right)}{2}-\frac{\mathcal{P}^{\eta} \Gamma(\eta+1)}{2\left(l_{2}^{\mathcal{P}}-l_{1}^{\mathcal{P}}\right)^{\eta}}\left[^{\mathcal{P}} \mathcal{I}_{l_{1}^{+}}^{\eta} \hbar\left(l_{2}\right)+{ }^{\mathcal{P}} \mathcal{I}_{l_{2}^{\eta}}^{\eta} \hbar\left(l_{1}\right)\right]\right| \\
& \leq \frac{l_{2}^{\mathcal{P}}-l_{1}^{\mathcal{P}}}{2 \mathcal{P}}\left[\left(\int_{0}^{1}(1-\xi)\left[(1-\xi)^{\eta}-\xi^{\eta}\right]\left(\xi l_{1}^{\mathcal{P}}+(1-\xi) l_{2}^{\mathcal{P}}\right)^{\left(\frac{1-\mathcal{P}}{\mathcal{P}}\right)} d \xi\right)^{1-1 / \alpha}\right. \\
& \quad \times\left(\int_{0}^{1}(1-\xi)\left[(1-\xi)^{\eta}-\xi^{\eta}\right]\right. \\
& \left.\quad \times\left(\xi l_{1}^{\mathcal{P}}+(1-\xi) l_{2}^{\mathcal{P}}\right)^{\left(\frac{1-\mathcal{P}}{\mathcal{P}}\right)}\left|\hbar^{\prime}\left({ }^{\mathcal{P}} \sqrt{\xi l_{1}^{\mathcal{P}}+(1-\xi) l_{2}^{\mathcal{P}}}\right)\right|^{\beta} d \xi\right)^{1 / \beta} \\
& \quad+\left(\int_{0}^{1} \xi\left[(1-\xi)^{\eta}-\xi^{\eta}\right]\left(\xi l_{1}^{\mathcal{P}}+(1-\xi) l_{2}^{\mathcal{P}}\right)^{\left(\frac{1-\mathcal{P}}{\mathcal{P}}\right)} d \xi\right)^{1-1 / \alpha} \\
& \quad \times\left(\int_{0}^{1} \xi\left[(1-\xi)^{\eta}-\xi^{\eta}\right]\right. \\
& \left.\left.\quad \times\left(\xi l_{1}^{\mathcal{P}}+(1-\xi) l_{2}^{\mathcal{P}}\right)^{\left(\frac{1-\mathcal{P}}{\mathcal{P}}\right)}\left|\hbar^{\prime}\left(\mathcal{P} \sqrt{\xi l_{1}^{\mathcal{P}}+(1-\xi) l_{2}^{\mathcal{P}}}\right)\right|^{\beta} d \xi\right)^{1 / \beta}\right]
\end{aligned}
$$


From the $n$-polynomial $\mathcal{P}$-convexity of the function $\left|\hbar^{\prime}\right|^{\beta}$ on $\Omega$, we have

$$
\begin{aligned}
& \int_{0}^{1}(1-\xi)\left[(1-\xi)^{\eta}-\xi^{\eta}\right]\left(\xi l_{1}^{\mathcal{P}}+(1-\xi) l_{2}^{\mathcal{P}}\right)^{\left(\frac{1-\mathcal{P}}{\mathcal{P}}\right)}\left|\hbar^{\prime}\left(\mathcal{P} \sqrt{\xi l_{1}^{\mathcal{P}}+(1-\xi) l_{2}^{\mathcal{P}}}\right)\right|^{\beta} d \xi \\
& \leq \int_{0}^{1}(1-\xi)\left[(1-\xi)^{\eta}-\xi^{\eta}\right]\left(\xi l_{1}^{\mathcal{P}}+(1-\xi) l_{2}^{\mathcal{P}}\right)^{\left(\frac{1-\mathcal{P}}{\mathcal{P}}\right)} \\
& \quad \times\left[\frac{1}{n} \sum_{\theta=1}^{n}\left[1-(1-\xi)^{\theta}\right]\left|\hbar\left(l_{1}\right)\right|^{\beta}+\frac{1}{n} \sum_{\theta=1}^{n}\left[1-\xi^{\theta}\right]\left|\hbar\left(l_{2}\right)\right|^{\beta}\right] d \xi \\
& \leq \frac{\left|\hbar\left(l_{1}\right)\right|^{\beta}}{n} \sum_{\theta=1}^{n}\left[\int_{0}^{1}\left(\xi l_{1}^{\mathcal{P}}+(1-\xi) l_{2}^{\mathcal{P}}\right)^{\left(\frac{1-\mathcal{P}}{\mathcal{P}}\right)}\right. \\
& \left.\quad \times\left[(1-\xi)^{\eta+1}-(1-\xi)^{\theta+\eta+1}-\xi^{\eta}(1-\xi)+\xi^{\eta}(1-\xi)^{\theta+1}\right]\right] d \xi \\
& \quad+\frac{\left|\hbar\left(l_{2}\right)\right|^{\beta}}{n} \sum_{\theta=1}^{n}\left[\int_{0}^{1}\left(\xi l_{1}^{\mathcal{P}}+(1-\xi) l_{2}^{\mathcal{P}}\right)^{\left(\frac{1-\mathcal{P}}{\mathcal{P}}\right)}\right. \\
& \left.\quad \times\left[(1-\xi)^{\eta+1}-\xi^{\theta}(1-\xi)^{\eta+1}-\xi^{\eta}(1-\xi)+\xi^{\theta+\eta}(1-\xi)\right]\right] d \xi \\
& =\frac{\left|\hbar\left(l_{1}\right)\right|^{\beta}}{n} \sum_{\theta=1}^{n} \Psi_{2}^{*}\left(l_{1}, l_{2} ; \mathcal{P}\right)+\frac{\left|\hbar\left(l_{2}\right)\right|^{\beta}}{n} \sum_{\theta=1}^{n} \Psi_{3}^{*}\left(l_{1}, l_{2} ; \mathcal{P}\right) .
\end{aligned}
$$

Similarly, we have

$$
\begin{aligned}
\int_{0}^{1} \xi & {\left[(1-\xi)^{\eta}-\xi^{\eta}\right]\left(\xi l_{1}^{\mathcal{P}}+(1-\xi) l_{2}^{\mathcal{P}}\right)^{\left(\frac{1-\mathcal{P}}{\mathcal{P}}\right)} \mid \hbar^{\prime}\left(\left.{ }^{\mathcal{P}} \sqrt{\left.\xi l_{1}^{\mathcal{P}}+(1-\xi) l_{2}^{\mathcal{P}}\right)}\right|^{\beta} d \xi\right.} \\
\leq & \int_{0}^{1}\left[\xi(1-\xi)^{\eta}-\xi^{\eta+1}\right]\left(\xi l_{1}^{\mathcal{P}}+(1-\xi) l_{2}^{\mathcal{P}}\right)^{\left(\frac{1-\mathcal{P}}{\mathcal{P}}\right)} \\
& \times\left[\frac{1}{n} \sum_{\theta=1}^{n}\left[1-(1-\xi)^{\theta}\right]\left|\hbar\left(l_{1}\right)\right|^{\beta}+\frac{1}{n} \sum_{\theta=1}^{n}\left[1-\xi^{\theta}\right]\left|\hbar\left(l_{2}\right)\right|^{\beta}\right] d \xi \\
\leq & \frac{\left|\hbar\left(l_{1}\right)\right|^{\beta}}{n} \sum_{\theta=1}^{n}\left[\int_{0}^{1}\left(\xi l_{1}^{\mathcal{P}}+(1-\xi) l_{2}^{\mathcal{P}}\right)^{\left(\frac{1-\mathcal{P}}{\mathcal{P}}\right)}\right. \\
& \left.\times\left[\xi(1-\xi)^{\eta}-\xi(1-\xi)^{\theta+\eta}-\xi^{\eta+1}+\xi^{\eta+1}(1-\xi)^{\theta}\right]\right] d \xi \\
& +\frac{\left|\hbar\left(l_{2}\right)\right|^{\beta}}{n} \sum_{\theta=1}^{n}\left[\int_{0}^{1}\left(\xi l_{1}^{\mathcal{P}}+(1-\xi) l_{2}^{\mathcal{P}}\right)^{\left(\frac{1-\mathcal{P}}{\mathcal{P}}\right)}\right. \\
& \left.\times\left[\xi(1-\xi)^{\eta}-\xi^{\theta+1}(1-\xi)^{\eta}-\xi^{\eta+1}+\xi^{\theta+\eta+1}\right]\right] d \xi \\
= & \frac{\left|\hbar\left(l_{1}\right)\right|^{\beta}}{n} \sum_{\theta=1}^{n} \Psi_{5}^{*}\left(l_{1}, l_{2} ; \mathcal{P}\right)+\frac{\left|\hbar\left(l_{2}\right)\right|^{\beta}}{n} \sum_{\theta=1}^{n} \Psi_{6}^{*}\left(l_{1}, l_{2} ; \mathcal{P}\right) .
\end{aligned}
$$

Inequalities (4.10)-(4.12) lead to

$$
\left|\frac{\hbar\left(l_{1}\right)+\hbar\left(l_{2}\right)}{2}-\frac{\mathcal{P}^{\eta} \Gamma(\eta+1)}{2\left(l_{2}^{\mathcal{P}}-l_{1}^{\mathcal{P}}\right)^{\eta}}\left[{ }^{\mathcal{P}} \mathcal{I}_{l_{1}^{+}}^{\eta} \hbar\left(l_{2}\right)+{ }^{\mathcal{P}} \mathcal{I}_{l_{2}^{-}}^{\eta} \hbar\left(l_{1}\right)\right]\right|
$$




$$
\begin{aligned}
\leq & \frac{l_{2}^{\mathcal{P}}-l_{1}^{\mathcal{P}}}{2 \mathcal{P}}\left[\left(\Psi_{1}^{*}\left(l_{1}, l_{2} ; \mathcal{P}\right)\right)^{1-1 / \alpha}\right. \\
& \times\left(\frac{\left|\hbar\left(l_{1}\right)\right|^{\beta}}{n} \sum_{\theta=1}^{n} \Psi_{2}^{*}\left(l_{1}, l_{2} ; \mathcal{P}\right)+\frac{\left|\hbar\left(l_{2}\right)\right|^{\beta}}{n} \sum_{\theta=1}^{n} \Psi_{3}^{*}\left(l_{1}, l_{2} ; \mathcal{P}\right)\right)^{1 / \beta} \\
& \left.+\left(\Psi_{4}^{*}\left(l_{1}, l_{2} ; \mathcal{P}\right)\right)^{1-1 / \alpha}\left(\frac{\left|\hbar\left(l_{1}\right)\right|^{\beta}}{n} \sum_{\theta=1}^{n} \Psi_{5}^{*}\left(l_{1}, l_{2} ; \mathcal{P}\right)+\frac{\left|\hbar\left(l_{2}\right)\right|^{\beta}}{n} \sum_{\theta=1}^{n} \Psi_{6}^{*}\left(l_{1}, l_{2} ; \mathcal{P}\right)\right)^{1 / \beta}\right],
\end{aligned}
$$

where we have used the facts that

$$
\begin{aligned}
& \Psi_{1}^{*}\left(l_{1}, l_{2} ; \mathcal{P}\right):=\int_{0}^{1}(1-\xi)\left[(1-\xi)^{\eta}-\xi^{\eta}\right]\left(\xi l_{1}^{\mathcal{P}}+(1-\xi) l_{2}^{\mathcal{P}}\right)^{\frac{1-\mathcal{P}}{\mathcal{P}}} d \xi \\
& =\frac{1}{l_{2}^{(\mathcal{P}-1)}}\left\{\begin{array}{c}
\frac{1}{\eta+2} 2 \mathcal{F}_{1}\left((1-1 / \mathcal{P}), 1, \eta+3,1-\left(l_{1} / l_{2}\right)^{\mathcal{P}}\right) \\
-\frac{1}{(\eta+2)(\eta+3)}{ }^{2} \mathcal{F}_{1}\left((1-1 / \mathcal{P}), \eta+1, \eta+3,1-\left(l_{1} / l_{2}\right)^{\mathcal{P}}\right),
\end{array}\right. \\
& \Psi_{2}^{*}\left(l_{1}, l_{2} ; \mathcal{P}\right):=\int_{0}^{1}\left(\xi l_{1}^{\mathcal{P}}+(1-\xi) l_{2}^{\mathcal{P}}\right)^{\frac{1-\mathcal{P}}{\mathcal{P}}} \\
& \times\left[(1-\xi)^{\eta+1}-(1-\xi)^{\theta+\eta+1}-\xi^{\eta}(1-\xi)+\xi^{\eta}(1-\xi)^{\theta+1}\right] d \xi \\
& =\frac{1}{l_{2}^{(\mathcal{P}-1)}}\left\{\begin{aligned}
\frac{1}{\eta+1} & {\left[{ }_{2} \mathcal{F}_{1}\left((1-1 / \mathcal{P}), 1, \eta+3,1-\left(l_{1} / l_{2}\right)^{\mathcal{P}}\right)\right.} \\
& \left.-\frac{1}{\eta+2} 2 \mathcal{F}_{1}\left((1-1 / \mathcal{P}), \eta+1, \eta+3,1-\left(l_{1} / l_{2}\right)^{\mathcal{P}}\right)\right] \\
& +\mathbb{B}(\eta+1, \theta+1) \\
& \times\left[{ }_{2} \mathcal{F}_{1}\left((1-1 / \mathcal{P}), \eta+1, \theta+\eta+3,1-\left(l_{1} / l_{2}\right)^{\mathcal{P}}\right)\right] \\
& -\frac{1}{\eta+\theta+1}\left[\mathcal{F}_{2}\left((1-1 / \mathcal{P}), 1, \theta+\eta+3,1-\left(l_{1} / l_{2}\right)^{\mathcal{P}}\right)\right],
\end{aligned}\right.
\end{aligned}
$$

and

$$
\begin{aligned}
\Psi_{3}^{*}\left(l_{1}, l_{2} ; \mathcal{P}\right):= & \int_{0}^{1}\left(\xi l_{1}^{\mathcal{P}}+(1-\xi) l_{2}^{\mathcal{P}}\right)^{\frac{1-\mathcal{P}}{\mathcal{P}}} \\
& \times\left[(1-\xi)^{\eta+1}-\xi^{\theta}(1-\xi)^{\eta+1}-\xi^{\eta}(1-\xi)+\xi^{\theta+\eta}(1-\xi)\right] d \xi \\
= & \frac{1}{l_{2}^{(\mathcal{P}-1)}}\left\{\begin{array}{c}
\frac{1}{\eta+1}\left[{ }_{2} \mathcal{F}_{1}\left((1-1 / \mathcal{P}), 1, \eta+3,1-\left(l_{1} / l_{2}\right)^{\mathcal{P}}\right)\right. \\
\left.-\frac{1}{\eta+2} 2 \mathcal{F}_{1}\left((1-1 / \mathcal{P}), \eta+1, \eta+3,1-\left(l_{1} / l_{2}\right)^{\mathcal{P}}\right)\right] \\
-\mathbb{B}(\eta+2, \theta+1) \\
\times\left[\mathcal{F}_{1}\left((1-1 / \mathcal{P}), \eta+1, \theta+\eta+3,1-\left(l_{1} / l_{2}\right)^{\mathcal{P}}\right)\right] \\
\\
+\frac{1}{\eta+\theta+1}\left[\mathcal{F}_{2}\left((1-1 / \mathcal{P}), \theta+\eta+1, \theta+\eta+3,1-\left(l_{1} / l_{2}\right)^{\mathcal{P}}\right)\right]
\end{array}\right.
\end{aligned}
$$

The desired inequality (4.9) can be obtained by adopting the same technique for integrals in (4.13) and substituting the obtained results together with the equalities (4.14) and (4.15) in (4.13).

\section{Ostrowski type inequalities}

This section is devoted to establishing novel bounds that refine the Ostrowski type inequality for mappings whose first derivative in absolute value at a certain power is a $n$ - 
polynomial $\mathcal{P}$-convex function. It is noteworthy that Thatsatian et al. [37] contemplated the following lemma for generalized fractional integrals.

Lemma 5.1 (See [37]) Let $\eta>0, \mathcal{P} \in \mathbb{R} \backslash\{0\}$ and $\hbar: \Omega \rightarrow \mathbb{R}$ be a differentiable function on $\Omega^{\circ}\left(\Omega^{\circ}\right.$ is the interior of $\left.\Omega\right)$ such that $l_{1}, l_{2} \in \Omega$ with $l_{1}<l_{2}$ and $\hbar^{\prime} \in L_{1}\left(\left[l_{1}, l_{2}\right]\right)$. Then we have the inequality

$$
\begin{aligned}
\frac{\left(z^{\mathcal{P}}-l_{1}^{\mathcal{P}}\right)^{\eta} \hbar\left(l_{1}\right)+\left(l_{2}^{\mathcal{P}}-z^{\mathcal{P}}\right)^{\eta} \hbar\left(l_{2}\right)}{\mathcal{P}^{\eta}\left(l_{2}-l_{1}\right)}-\frac{\Gamma(\eta+1)}{l_{2}-l_{1}}\left[\left({ }^{\mathcal{P}} \mathcal{I}_{l_{1}^{\dagger}}^{\eta} \hbar\right)(z)+\left({ }^{\mathcal{P}} \mathcal{I}_{l_{2}^{\eta}}^{\eta} \hbar\right)(z)\right] \\
=-\frac{\left(z^{\mathcal{P}}-l_{1}^{\mathcal{P}}\right)^{\eta+1}}{\mathcal{P}^{1+\eta}\left(l_{2}-l_{1}\right)} \int_{0}^{1} \xi^{\eta}\left(\xi l_{1}^{\mathcal{P}}+(1-\xi) z^{\mathcal{P}}\right)^{\frac{1-\mathcal{P}}{\mathcal{P}}}\left|\hbar^{\prime}\left({ }^{\mathcal{P}} \sqrt{\xi l_{1}^{\mathcal{P}}+(1-\xi) z^{\mathcal{P}}}\right)\right| d \xi \\
\quad+\frac{\left(l_{2}^{\mathcal{P}}-z^{\mathcal{P}}\right)^{\eta+1}}{\mathcal{P}^{1+\eta}\left(l_{2}-l_{1}\right)} \int_{0}^{1} \xi^{\eta}\left(\xi l_{2}^{\mathcal{P}}+(1-\xi) z^{\mathcal{P}}\right)^{\frac{1-\mathcal{P}}{\mathcal{P}}}\left|\hbar^{\prime}\left(\mathcal{P} \sqrt{\xi l_{2}^{\mathcal{P}}+(1-\xi) z^{\mathcal{P}}}\right)\right| d \xi .
\end{aligned}
$$

Theorem 5.2 Let $n \in \mathbb{N}, \eta>0, l_{1}, l_{2} \in \Omega$ with $l_{1}<l_{2}$, and $\hbar: \Omega \subset(0, \infty) \rightarrow \mathbb{R}$ be a differentiable function on $\Omega^{\circ}$ such that $\hbar^{\prime} \in L_{1}\left(\left[l_{1}, l_{2}\right]\right)$ and $\left|\hbar^{\prime}\right|$ is a n-polynomial $\mathcal{P}$-convex function satisfies $\left|\hbar^{\prime}(z)\right| \leq \mathcal{M}$ for all $z \in\left[l_{1}, l_{2}\right]$. Then the inequality

$$
\begin{aligned}
& \left|\frac{\left(z^{\mathcal{P}}-l_{1}^{\mathcal{P}}\right)^{\eta} \hbar\left(l_{1}\right)+\left(l_{2}^{\mathcal{P}}-z^{\mathcal{P}}\right)^{\eta} \hbar\left(l_{2}\right)}{\mathcal{P}^{\eta}\left(l_{2}-l_{1}\right)}-\frac{\Gamma(\eta+1)}{l_{2}-l_{1}}\left[\left({ }^{\mathcal{P}} \mathcal{I}_{l_{1}^{+}}^{\eta} \hbar\right)(z)+\left({ }^{\mathcal{P}} \mathcal{I}_{l_{2}^{-}}^{\eta} \hbar\right)(z)\right]\right| \\
& \quad \leq \frac{l_{1}^{1-\mathcal{P}} \mathcal{M}}{\mathcal{P}^{1+\eta}}\left[\frac{\left(z^{\mathcal{P}}-l_{1}^{\mathcal{P}}\right)^{\eta+1}+\left(l_{2}^{\mathcal{P}}-z^{\mathcal{P}}\right)^{\eta+1}}{\left(l_{2}-l_{1}\right)}\right] \frac{1}{n} \sum_{\theta=1}^{n}\left[\frac{\eta+2 \theta+1}{(\eta+1)(\eta+\theta+1)}-\mathbb{B}(\eta-1, \theta-1)\right]
\end{aligned}
$$

holds for all $z \in\left(l_{1}, l_{2}\right)$ and $\mathcal{P} \in(1, \infty)$, and the inequality

$$
\begin{aligned}
& \left|\frac{\left(z^{\mathcal{P}}-l_{1}^{\mathcal{P}}\right)^{\eta} \hbar\left(l_{1}\right)+\left(l_{2}^{\mathcal{P}}-z^{\mathcal{P}}\right)^{\eta} \hbar\left(l_{2}\right)}{\mathcal{P}^{\eta}\left(l_{2}-l_{1}\right)}-\frac{\Gamma(\eta+1)}{l_{2}-l_{1}}\left[\left({ }^{\mathcal{P}} \mathcal{I}_{l_{1}^{+}}^{\eta} \hbar\right)(z)+\left({ }^{\mathcal{P}} \mathcal{I}_{l_{2}^{-}}^{\eta} \hbar\right)(z)\right]\right| \\
& \quad \leq \frac{l_{2}^{1-\mathcal{P}} \mathcal{M}}{\mathcal{P}^{1+\eta}}\left[\frac{\left(z^{\mathcal{P}}-l_{1}^{\mathcal{P}}\right)^{\eta+1}+\left(l_{2}^{\mathcal{P}}-z^{\mathcal{P}}\right)^{\eta+1}}{\left(l_{2}-l_{1}\right)}\right] \frac{1}{n} \sum_{\theta=1}^{n}\left[\frac{\eta+2 \theta+1}{(\eta+1)(\eta+\theta+1)}-\mathbb{B}(\eta-1, \theta-1)\right]
\end{aligned}
$$

holds for all $z \in\left(l_{1}, l_{2}\right)$ and $\mathcal{P} \in(-\infty, 0) \cup(0,1)$.

Proof To prove the first inequality of Theorem 5.2, we use Lemma 5.1 and the $n$ polynomial $\mathcal{P}$-convexity of $\left|\hbar^{\prime}\right|$ to get

$$
\begin{aligned}
& \left|\frac{\left(z^{\mathcal{P}}-l_{1}^{\mathcal{P}}\right)^{\eta} \hbar\left(l_{1}\right)+\left(l_{2}^{\mathcal{P}}-z^{\mathcal{P}}\right)^{\eta} \hbar\left(l_{2}\right)}{\mathcal{P}^{\eta}\left(l_{2}-l_{1}\right)}-\frac{\Gamma(\eta+1)}{l_{2}-l_{1}}\left[\left({ }^{\mathcal{P}} \mathcal{I}_{l_{1}^{\dagger}}^{\eta} \hbar\right)(z)+\left({ }^{\mathcal{P}} \mathcal{I}_{l_{2}^{-}}^{\eta} \hbar\right)(z)\right]\right| \\
& \leq \frac{\left(z^{\mathcal{P}}-l_{1}^{\mathcal{P}}\right)^{\eta+1}}{\mathcal{P}^{1+\eta}\left(l_{2}-l_{1}\right)} \int_{0}^{1} \xi^{\eta}\left(\xi l_{1}^{\mathcal{P}}+(1-\xi) z^{\mathcal{P}}\right)^{\frac{1-\mathcal{P}}{\mathcal{P}}}\left|\hbar^{\prime}\left(\mathcal{P} \sqrt{\xi l_{1}^{\mathcal{P}}+(1-\xi) z^{\mathcal{P}}}\right)\right| d \xi \\
& \quad+\frac{\left(l_{2}^{\mathcal{P}}-z^{\mathcal{P}}\right)^{\eta+1}}{\mathcal{P}^{1+\eta}\left(l_{2}-l_{1}\right)} \int_{0}^{1} \xi^{\eta}\left(\xi l_{2}^{\mathcal{P}}+(1-\xi) z^{\mathcal{P}}\right)^{\frac{1-\mathcal{P}}{\mathcal{P}}}\left|\hbar^{\prime}\left(\mathcal{P} \sqrt{\xi l_{2}^{\mathcal{P}}+(1-\xi) z^{\mathcal{P}}}\right)\right| d \xi \\
& \leq \frac{\left(z^{\mathcal{P}}-l_{1}^{\mathcal{P}}\right)^{\eta+1}}{\mathcal{P}^{1+\eta}\left(l_{2}-l_{1}\right)} \int_{0}^{1} \xi^{\eta}\left(\xi l_{1}^{\mathcal{P}}+(1-\xi) z^{\mathcal{P}}\right)^{\frac{1-\mathcal{P}}{\mathcal{P}}} \\
& \left.\quad \times\left[\frac{1}{n} \sum_{\theta=1}^{n}\left[1-(1-\xi)^{\theta}\right]\left|\hbar^{\prime}\left(l_{1}\right)\right|+\frac{1}{n} \sum_{\theta=1}^{n}\left[1-\xi^{\theta}\right] \mid \hbar^{\prime}(z)\right]\right] d \xi
\end{aligned}
$$




$$
\begin{aligned}
& +\frac{\left(l_{2}^{\mathcal{P}}-z^{\mathcal{P}}\right)^{\eta+1}}{\mathcal{P}^{1+\eta}\left(l_{2}-l_{1}\right)} \int_{0}^{1} \xi^{\eta}\left(\xi l_{2}^{\mathcal{P}}+(1-\xi) z^{\mathcal{P}}\right)^{\frac{1-\mathcal{P}}{\mathcal{P}}} \\
& \times\left[\frac{1}{n} \sum_{\theta=1}^{n}\left[1-(1-\xi)^{\theta}\right]\left|\hbar^{\prime}\left(l_{2}\right)\right|+\frac{1}{n} \sum_{\theta=1}^{n}\left[1-\xi^{\theta}\right]\left|\hbar^{\prime}(z)\right|\right] d \xi .
\end{aligned}
$$

Since $\mathcal{P} \in(1, \infty)$, we conclude that

$$
\left(\xi l_{2}^{\mathcal{P}}+(1-\xi) z^{\mathcal{P}}\right)^{\frac{1-\mathcal{P}}{\mathcal{P}}} \leq\left(\xi l_{1}^{\mathcal{P}}+(1-\xi) z^{\mathcal{P}}\right)^{\frac{1-\mathcal{P}}{\mathcal{P}}} \leq l_{1}^{1-\mathcal{P}}
$$

by simple computations, we have

$$
\begin{array}{r}
\int_{0}^{1} \xi^{\eta}\left[\frac{1}{n} \sum_{\theta=1}^{n}\left[1-(1-\xi)^{\theta}\right]+\frac{1}{n} \sum_{\theta=1}^{n}\left[1-\xi^{\theta}\right]\right] d \xi \\
=\frac{1}{n} \sum_{\theta=1}^{n}\left[\frac{\eta+2 \theta+1}{(\eta+1)(\eta+\theta+1)}-\mathbb{B}(\eta-1, \theta-1)\right],
\end{array}
$$

which implies first inequality of Theorem 5.2.

To prove the second inequality of Theorem 5.2 , we use $\mathcal{P} \in(-\infty, 0) \cup(0,1)$ to get

$$
\left(\xi l_{1}^{\mathcal{P}}+(1-\xi) z^{\mathcal{P}}\right)^{\frac{1-\mathcal{P}}{\mathcal{P}}} \leq\left(\xi l_{2}^{\mathcal{P}}+(1-\xi) z^{\mathcal{P}}\right)^{\frac{1-\mathcal{P}}{\mathcal{P}}} \leq l_{2}^{1-\mathcal{P}},
$$

which completes the second inequality of Theorem 5.2.

Theorem 5.3 Let $n \in \mathbb{N}, \alpha, \beta>1$ with $\alpha^{-1}+\beta^{-1}=1, l_{1}, l_{2} \in \Omega$ with $l_{1}<l_{2}$, and $\hbar: \Omega \subset$ $(0, \infty) \rightarrow \mathbb{R}$ be a differentiable function on $\Omega^{\circ}$ such that $\hbar^{\prime} \in L_{1}\left(\left[l_{1}, l_{2}\right]\right)$ and $\left|\hbar^{\prime}\right|^{\beta}$ is a $n$ polynomial convex function satisfies $\left|\hbar^{\prime}(z)\right| \leq \mathcal{M}$ for all $z \in\left[l_{1}, l_{2}\right]$. Then the inequality

$$
\begin{gathered}
\left|\frac{\left(z^{\mathcal{P}}-l_{1}^{\mathcal{P}}\right)^{\eta} \hbar\left(l_{1}\right)+\left(l_{2}^{\mathcal{P}}-z^{\mathcal{P}}\right)^{\eta} \hbar\left(l_{2}\right)}{\mathcal{P}^{\eta}\left(l_{2}-l_{1}\right)}-\frac{\Gamma(\eta+1)}{l_{2}-l_{1}}\left[\left({ }^{\mathcal{P}} \mathcal{I}_{l_{1}^{+}}^{\eta} \hbar\right)(z)+\left({ }^{\mathcal{P}} \mathcal{I}_{l_{2}}^{\eta} \hbar\right)(z)\right]\right| \\
\leq \frac{l_{1}^{1-\mathcal{P}} \mathcal{M}}{\mathcal{P}^{1+\eta}(1+\alpha \eta)^{1 / \alpha}}\left[\frac{\left(z^{\mathcal{P}}-l_{1}^{\mathcal{P}}\right)^{\eta+1}+\left(l_{2}^{\mathcal{P}}-z^{\mathcal{P}}\right)^{\eta+1}}{\left(l_{2}-l_{1}\right)}\right]\left(\frac{1}{n} \sum_{\theta=1}^{n} \frac{2 \theta}{\theta+1}\right)^{1 / \beta}
\end{gathered}
$$

holds for all $z \in\left(l_{1}, l_{2}\right)$ and $\mathcal{P} \in(1, \infty)$, and the inequality

$$
\begin{gathered}
\left|\frac{\left(z^{\mathcal{P}}-l_{1}^{\mathcal{P}}\right)^{\eta} \hbar\left(l_{1}\right)+\left(l_{2}^{\mathcal{P}}-z^{\mathcal{P}}\right)^{\eta} \hbar\left(l_{2}\right)}{\mathcal{P}^{\eta}\left(l_{2}-l_{1}\right)}-\frac{\Gamma(\eta+1)}{l_{2}-l_{1}}\left[\left({ }^{\mathcal{P}} \mathcal{I}_{l_{1}^{+}}^{\eta} \hbar\right)(z)+\left({ }^{\mathcal{P}} \mathcal{I}_{l_{2}}^{\eta} \hbar\right)(z)\right]\right| \\
\leq \frac{l_{2}^{1-\mathcal{P}} \mathcal{M}}{\mathcal{P}^{1+\eta}(1+\alpha \eta)^{1 / \alpha}}\left[\frac{\left(z^{\mathcal{P}}-l_{1}^{\mathcal{P}}\right)^{\eta+1}+\left(l_{2}^{\mathcal{P}}-z^{\mathcal{P}}\right)^{\eta+1}}{\left(l_{2}-l_{1}\right)}\right]\left(\frac{1}{n} \sum_{\theta=1}^{n} \frac{2 \theta}{\theta+1}\right)^{1 / \beta}
\end{gathered}
$$

holds for all $z \in\left(l_{1}, l_{2}\right)$ and $\mathcal{P} \in(-\infty, 0) \cup(0,1)$. 
Proof To prove first inequality of Theorem 5.3, we use Lemma 5.1, (5.5) and the Hölder inequality to obtain

$$
\begin{aligned}
& \mid \frac{\left(z^{\mathcal{P}}-l_{1}^{\mathcal{P}}\right)^{\eta} \hbar\left(l_{1}\right)+\left(l_{2}^{\mathcal{P}}-z^{\mathcal{P}}\right)^{\eta} \hbar\left(l_{2}\right)}{\mathcal{P}^{\eta}\left(l_{2}-l_{1}\right)}-\frac{\Gamma(\eta+1)}{l_{2}-l_{1}}\left[\left({ }^{\mathcal{P}} \mathcal{I}_{l_{1}^{+}}^{\eta} \hbar\right)(z)+\left({ }^{\mathcal{P}} \mathcal{I}_{l_{2}^{-}}^{\eta} \hbar\right)(z)\right] \mid \\
& \leq \frac{\left(z^{\mathcal{P}}-l_{1}^{\mathcal{P}}\right)^{\eta+1}}{\mathcal{P}^{1+\eta}\left(l_{2}-l_{1}\right)} \int_{0}^{1} \xi^{\eta}\left(\xi l_{1}^{\mathcal{P}}+(1-\xi) z^{\mathcal{P}}\right)^{\frac{1-\mathcal{P}}{\mathcal{P}}}\left|\hbar^{\prime}\left(\mathcal{P} \sqrt{\xi l_{1}^{\mathcal{P}}+(1-\xi) z^{\mathcal{P}}}\right)\right| d \xi \\
& \quad+\frac{\left(l_{2}^{\mathcal{P}}-z^{\mathcal{P}}\right)^{\eta+1}}{\mathcal{P}^{1+\eta}\left(l_{2}-l_{1}\right)} \int_{0}^{1} \xi^{\eta}\left(\xi l_{2}^{\mathcal{P}}+(1-\xi) z^{\mathcal{P}}\right)^{\frac{1-\mathcal{P}}{\mathcal{P}}}\left|\hbar^{\prime}\left(\mathcal{P} \sqrt{\xi l_{2}^{\mathcal{P}}+(1-\xi) z^{\mathcal{P}}}\right)\right| d \xi \\
& \leq \frac{l_{1}^{1-\mathcal{P}}\left(z^{\mathcal{P}}-l_{1}^{\mathcal{P}}\right)^{\eta+1}}{\mathcal{P}^{1+\eta}\left(l_{2}-l_{1}\right)} \int_{0}^{1} \xi^{\eta}\left(\xi l_{1}^{\mathcal{P}}+(1-\xi) z^{\mathcal{P}}\right)^{\frac{1-\mathcal{P}}{\mathcal{P}}}\left|\hbar^{\prime}\left({ }^{\mathcal{P}} \sqrt{\xi l_{1}^{\mathcal{P}}+(1-\xi) z^{\mathcal{P}}}\right)\right| d \xi \\
& \quad+\frac{l_{2}^{1-\mathcal{P}}\left(l_{2}^{\mathcal{P}}-z^{\mathcal{P}}\right)^{\eta+1}}{\mathcal{P}^{1+\eta}\left(l_{2}-l_{1}\right)} \int_{0}^{1} \xi^{\eta}\left(\xi l_{2}^{\mathcal{P}}+(1-\xi) z^{\mathcal{P}}\right)^{\frac{1-\mathcal{P}}{\mathcal{P}}}\left|\hbar^{\prime}\left(\mathcal{P} \sqrt{\xi l_{2}^{\mathcal{P}}+(1-\xi) z^{\mathcal{P}}}\right)\right| d \xi \\
& \leq \frac{l_{1}^{1-\mathcal{P}}\left(z^{\mathcal{P}}-l_{1}^{\mathcal{P}}\right)^{\eta+1}}{\mathcal{P}^{1+\eta}\left(l_{2}-l_{1}\right)}\left(\int_{0}^{1} \xi^{\alpha \eta} d \xi\right)^{1 / \alpha}\left(\int_{0}^{1}\left|\hbar^{\prime}\left({ }^{\mathcal{P}} \sqrt{\xi l_{1}^{\mathcal{P}}+(1-\xi) z^{\mathcal{P}}}\right)\right|^{\beta} d \xi\right)^{1 / \beta} \\
& \quad+\frac{l_{2}^{1-\mathcal{P}}\left(l_{2}^{\mathcal{P}}-z^{\mathcal{P}}\right)^{\eta+1}}{\mathcal{P}^{1+\eta}\left(l_{2}-l_{1}\right)}\left(\int_{0}^{1} \xi^{\alpha \eta} d \xi\right)^{1 / \alpha}\left(\int_{0}^{1}\left|\hbar^{\prime}\left(\mathcal{P}^{\mathcal{P}} \sqrt{\xi l_{2}^{\mathcal{P}}+(1-\xi) z^{\mathcal{P}}}\right)\right|^{\beta} d \xi\right)^{1 / \beta} .
\end{aligned}
$$

Since $\left|\hbar^{\prime}\right|^{\beta}$ is $n$-polynomial $\mathcal{P}$-convex and $\left|\hbar^{\prime}(z)\right| \leq \mathcal{M}$ for all $z \in\left[l_{1}, l_{2}\right]$, we get

$$
\begin{aligned}
& \int_{0}^{1}\left|\hbar^{\prime}\left(\sqrt[\mathcal{P}]{\xi l_{1}^{\mathcal{P}}+(1-\xi) z^{\mathcal{P}}}\right)\right|^{\beta} d \xi \\
& \quad \leq \int_{0}^{1}\left[\frac{1}{n} \sum_{\theta=1}^{n}\left[1-(1-\xi)^{\theta}\right]\left|\hbar^{\prime}\left(l_{1}\right)\right|^{\beta}+\frac{1}{n} \sum_{\theta=1}^{n}\left[1-\xi^{\theta}\right]\left|\hbar^{\prime}(z)\right|^{\beta}\right] d \xi \\
& \quad \leq \frac{\mathcal{M}^{\beta}}{n} \sum_{\theta=1}^{n} \int_{0}^{1}\left[2-(1-\xi)^{\theta}-\xi^{\theta}\right] d \xi \\
& \leq \frac{\mathcal{M}^{\beta}}{n} \sum_{\theta=1}^{n} \frac{2 \theta}{\theta+1}
\end{aligned}
$$

and

$$
\int_{0}^{1}\left|\hbar^{\prime}\left(\mathcal{P} \sqrt{\xi l_{2}^{\mathcal{P}}+(1-\xi) z^{\mathcal{P}}}\right)\right|^{\beta} d \xi \leq \frac{\mathcal{M}^{\beta}}{n} \sum_{\theta=1}^{n} \frac{2 \theta}{\theta+1} .
$$

Note that

$$
\int_{0}^{1} \xi^{\alpha \eta} d \xi=\frac{1}{\alpha \eta+1}
$$

Combining all above inequalities we get the first inequality of Theorem 5.3. The second inequality of Theorem 5.3 can be proved in a similar way and using (5.6).

Theorem 5.4 Let $n \in \mathbb{N}, \alpha, \beta>1$ with $\alpha^{-1}+\beta^{-1}=1, l_{1}, l_{2} \in \Omega$ with $l_{1}<l_{2}$, and $\hbar: \Omega \subset$ $(0, \infty) \rightarrow \mathbb{R}$ be a differentiable function on $\Omega^{\circ}$ such that $\hbar^{\prime} \in L_{1}\left(\left[l_{1}, l_{2}\right]\right)$ and $\left|\hbar^{\prime}\right|^{\beta}$ is a $n$ - 
polynomial $\mathcal{P}$-convex function satisfies $\left|\hbar^{\prime}(z)\right| \leq \mathcal{M}$ for all $z \in\left[l_{1}, l_{2}\right]$. Then the inequality

$$
\begin{aligned}
& \left|\frac{\left(z^{\mathcal{P}}-l_{1}^{\mathcal{P}}\right)^{\eta} \hbar\left(l_{1}\right)+\left(l_{2}^{\mathcal{P}}-z^{\mathcal{P}}\right)^{\eta} \hbar\left(l_{2}\right)}{\mathcal{P}^{\eta}\left(l_{2}-l_{1}\right)}-\frac{\Gamma(\eta+1)}{l_{2}-l_{1}}\left[\left({ }^{\mathcal{P}} \mathcal{I}_{l_{1}^{+}}^{\eta} \hbar\right)(z)+\left({ }^{\mathcal{P}} \mathcal{I}_{l_{2}^{\eta}}^{\eta} \hbar\right)(z)\right]\right| \\
& \leq \frac{l_{1}^{1-\mathcal{P}} \mathcal{M}}{\mathcal{P}^{1+\eta}(1+\alpha \eta)^{1 / \alpha}}\left[\frac{\left(z^{\mathcal{P}}-l_{1}^{\mathcal{P}}\right)^{\eta+1}+\left(l_{2}^{\mathcal{P}}-z^{\mathcal{P}}\right)^{\eta+1}}{\left(l_{2}-l_{1}\right)}\right] \\
& \quad \times\left(\frac{\mathcal{M}^{\beta}}{n} \sum_{\theta=1}^{n}\left[\frac{\beta \eta+2 \theta+1}{(\eta \beta+1)(\eta \beta+\theta+1)}-\mathbb{B}(\theta+1, \beta \eta+1)\right]\right)^{1 / \beta}
\end{aligned}
$$

holds for all $z \in\left(l_{1}, l_{2}\right)$ and $\mathcal{P} \in(1, \infty)$, and the inequality

$$
\begin{aligned}
& \left|\frac{\left(z^{\mathcal{P}}-l_{1}^{\mathcal{P}}\right)^{\eta} \hbar\left(l_{1}\right)+\left(l_{2}^{\mathcal{P}}-z^{\mathcal{P}}\right)^{\eta} \hbar\left(l_{2}\right)}{\mathcal{P}^{\eta}\left(l_{2}-l_{1}\right)}-\frac{\Gamma(\eta+1)}{l_{2}-l_{1}}\left[\left({ }^{\mathcal{P}} \mathcal{I}_{l_{1}^{+}}^{\eta} \hbar\right)(z)+\left({ }^{\mathcal{P}} \mathcal{I}_{l_{2}}^{\eta} \hbar\right)(z)\right]\right| \\
& \leq \frac{l_{2}^{1-\mathcal{P}} \mathcal{M}}{\mathcal{P}^{1+\eta}(1+\alpha \eta)^{1 / \alpha}}\left[\frac{\left(z^{\mathcal{P}}-l_{1}^{\mathcal{P}}\right)^{\eta+1}+\left(l_{2}^{\mathcal{P}}-z^{\mathcal{P}}\right)^{\eta+1}}{\left(l_{2}-l_{1}\right)}\right] \\
& \quad \times\left(\frac{\mathcal{M}^{\beta}}{n} \sum_{\theta=1}^{n}\left[\frac{\beta \eta+2 \theta+1}{(\eta \beta+1)(\eta \beta+\theta+1)}-\mathbb{B}(\theta+1, \beta \eta+1)\right]\right)^{1 / \beta}
\end{aligned}
$$

holds for all $z \in\left(l_{1}, l_{2}\right)$ and $\mathcal{P} \in(-\infty, 0) \cup(0,1)$.

Proof To prove first inequality of Theorem 5.4, we use Lemma 5.1, (5.5) and the powermean inequality to get

$$
\begin{aligned}
\left|\frac{\left(\mathcal{z}^{\mathcal{P}}-l_{1}^{\mathcal{P}}\right)^{\eta} \hbar\left(l_{1}\right)+\left(l_{2}^{\mathcal{P}}-z^{\mathcal{P}}\right)^{\eta} \hbar\left(l_{2}\right)}{\mathcal{P}^{\eta}\left(l_{2}-l_{1}\right)}-\frac{\Gamma(\eta+1)}{l_{2}-l_{1}}\left[\left({ }^{\mathcal{P}} \mathcal{I}_{l_{1}^{\dagger}}^{\eta} \hbar\right)(z)+\left({ }^{\mathcal{P}} \mathcal{I}_{l^{-}}^{\eta} \hbar\right)(z)\right]\right| \\
\leq \frac{\left(z^{\mathcal{P}}-l_{1}^{\mathcal{P}}\right)^{\eta+1}}{\mathcal{P}^{1+\eta}\left(l_{2}-l_{1}\right)} \int_{0}^{1} \xi^{\eta}\left(\xi l_{1}^{\mathcal{P}}+(1-\xi) z^{\mathcal{P}}\right)^{\frac{1-\mathcal{P}}{\mathcal{P}}}\left|\hbar^{\prime}\left(\mathcal{P} \sqrt{\xi l_{1}^{\mathcal{P}}+(1-\xi) z^{\mathcal{P}}}\right)\right| d \xi \\
\quad+\frac{\left(l_{2}^{\mathcal{P}}-z^{\mathcal{P}}\right)^{\eta+1}}{\mathcal{P}^{1+\eta}\left(l_{2}-l_{1}\right)} \int_{0}^{1} \xi^{\eta}\left(\xi l_{2}^{\mathcal{P}}+(1-\xi) z^{\mathcal{P}}\right)^{\frac{1-\mathcal{P}}{\mathcal{P}}}\left|\hbar^{\prime}\left(\mathcal{P} \sqrt{\xi l_{2}^{\mathcal{P}}+(1-\xi) z^{\mathcal{P}}}\right)\right| d \xi \\
\leq \frac{l_{1}^{1-\mathcal{P}}\left(z^{\mathcal{P}}-l_{1}^{\mathcal{P}}\right)^{\eta+1}}{\mathcal{P}^{1+\eta}\left(l_{2}-l_{1}\right)} \int_{0}^{1} \xi^{\eta}\left|\hbar^{\prime}\left({ }^{\mathcal{P}} \sqrt{\xi l_{1}^{\mathcal{P}}+(1-\xi) z^{\mathcal{P}}}\right)\right| d \xi \\
\quad+\frac{l_{2}^{1-\mathcal{P}}\left(l_{2}^{\mathcal{P}}-z^{\mathcal{P}}\right)^{\eta+1}}{\mathcal{P}^{1+\eta}\left(l_{2}-l_{1}\right)} \int_{0}^{1} \xi^{\eta}\left|\hbar^{\prime}\left(\mathcal{P} \sqrt{\xi l_{2}^{\mathcal{P}}+(1-\xi) z^{\mathcal{P}}}\right)\right| d \xi \\
\leq \frac{l_{1}^{1-\mathcal{P}}\left(z^{\mathcal{P}}-l_{1}^{\mathcal{P}}\right)^{\eta+1}}{\mathcal{P}^{1+\eta}\left(l_{2}-l_{1}\right)}\left(\int_{0}^{1} \xi^{\beta \eta}\left|\hbar^{\prime}\left({ }^{\mathcal{P}} \sqrt{\xi l_{1}^{\mathcal{P}}+(1-\xi) z^{\mathcal{P}}}\right)\right|^{\beta} d \xi\right)^{1 / \beta} \\
\quad+\frac{l_{2}^{1-\mathcal{P}}\left(l_{2}^{\mathcal{P}}-z^{\mathcal{P}}\right)^{\eta+1}}{\mathcal{P}^{1+\eta}\left(l_{2}-l_{1}\right)}\left(\int_{0}^{1} \xi^{\beta \eta}\left|\hbar^{\prime}\left(\mathcal{P} \sqrt{\xi l_{2}^{\mathcal{P}}+(1-\xi) z^{\mathcal{P}}}\right)\right|^{\beta} d \xi\right)^{1 / \beta} .
\end{aligned}
$$

Since $|\hbar|^{\beta}$ is $n$-polynomial $\mathcal{P}$-convex and $\left|\hbar^{\prime}(z)\right| \leq \mathcal{M}$ for all $z \in\left[l_{1}, l_{2}\right]$, we get

$$
\begin{aligned}
& \int_{0}^{1} \xi^{\beta \eta}\left|\hbar^{\prime}\left(\mathcal{P} \sqrt{\xi l_{1}^{\mathcal{P}}+(1-\xi) z^{\mathcal{P}}}\right)\right|^{\beta} d \xi \\
& \leq \int_{0}^{1} \xi^{\beta \eta}\left[\frac{1}{n} \sum_{\theta=1}^{n}\left[1-(1-\xi)^{\theta}\right]\left|\hbar^{\prime}\left(l_{1}\right)\right|^{\beta}+\frac{1}{n} \sum_{\theta=1}^{n}\left[1-\xi^{\theta}\right]\left|\hbar^{\prime}(z)\right|^{\beta}\right] d \xi
\end{aligned}
$$




$$
\begin{aligned}
& =\frac{\mathcal{M}^{\beta}}{n} \sum_{\theta=1}^{n} \int_{0}^{1}\left[2 \xi^{\eta \beta}-\xi^{\eta \beta}(1-\xi)^{\theta}+\xi^{\beta \eta}\left(1-\xi^{\theta}\right)\right] d \xi \\
& \leq \frac{\mathcal{M}^{\beta}}{n} \sum_{\theta=1}^{n}\left[\frac{\beta \eta+2 \theta+1}{(\eta \beta+1)(\eta \beta+\theta+1)}-\mathbb{B}(\theta+1, \beta \eta+1)\right] .
\end{aligned}
$$

Analogously,

$$
\begin{aligned}
& \int_{0}^{1} \xi^{\beta \eta}\left|\hbar^{\prime}\left(\mathcal{P} \sqrt{\xi l_{2}^{\mathcal{P}}+(1-\xi) \mathcal{z}^{\mathcal{P}}}\right)\right|^{\beta} d \xi \\
& \quad \leq \frac{\mathcal{M}^{\beta}}{n} \sum_{\theta=1}^{n}\left[\frac{\beta \eta+2 \theta+1}{(\eta \beta+1)(\eta \beta+\theta+1)}-\mathbb{B}(\theta+1, \beta \eta+1)\right] .
\end{aligned}
$$

Combining all above inequalities we get the first inequality of Theorem 5.4. The second inequality of Theorem 5.4 can be proved in a similar way by the use of (5.6).

Theorem 5.5 Let $n \in \mathbb{N}, \alpha, \beta>1$ with $\alpha^{-1}+\beta^{-1}=1, l_{1}, l_{2} \in \Omega$ with $l_{1}<l_{2}$, and $\hbar: \Omega \subset$ $(0, \infty) \rightarrow \mathbb{R}$ be a differentiable function on $\Omega^{\circ}$ such that $\hbar^{\prime} \in L_{1}\left(\left[l_{1}, l_{2}\right]\right)$ and $\left|\hbar^{\prime}\right|^{\beta}$ is a $n$ polynomial $\mathcal{P}$-convex function satisfies $\left|\hbar^{\prime}(z)\right| \leq \mathcal{M}$ for all $z \in\left[l_{1}, l_{2}\right]$. Then the inequality

$$
\begin{gathered}
\left|\frac{\left(z^{\mathcal{P}}-l_{1}^{\mathcal{P}}\right)^{\eta} \hbar\left(l_{1}\right)+\left(l_{2}^{\mathcal{P}}-z^{\mathcal{P}}\right)^{\eta} \hbar\left(l_{2}\right)}{\mathcal{P}^{\eta}\left(l_{2}-l_{1}\right)}-\frac{\Gamma(\eta+1)}{l_{2}-l_{1}}\left[\left({ }^{\mathcal{P}} \mathcal{I}_{l_{1}^{+}}^{\eta} \hbar\right)(z)+\left({ }^{\mathcal{P}} \mathcal{I}_{l_{2}^{\eta}}^{\eta} \hbar\right)(z)\right]\right| \\
\leq \frac{\left(z^{\mathcal{P}}-l_{1}^{\mathcal{P}}\right)^{\eta+1}+\left(l_{2}^{\mathcal{P}}-z^{\mathcal{P}}\right)^{\eta+1}}{\mathcal{P}^{1+\eta}\left(l_{2}-l_{1}\right)}\left[\frac{\left(l_{1}^{\alpha(1-\mathcal{P})}\right)}{\alpha(\alpha \eta+1)}+\frac{1}{\beta}\left(\frac{\mathcal{M}}{n} \sum_{\theta=1}^{n} \frac{2 \theta}{\theta+1}\right)^{\beta}\right]
\end{gathered}
$$

holds for all $z \in\left(l_{1}, l_{2}\right)$ and $\mathcal{P} \in(1, \infty)$, and the inequality

$$
\begin{gathered}
\left|\frac{\left(z^{\mathcal{P}}-l_{1}^{\mathcal{P}}\right)^{\eta} \hbar\left(l_{1}\right)+\left(l_{2}^{\mathcal{P}}-z^{\mathcal{P}}\right)^{\eta} \hbar\left(l_{2}\right)}{\mathcal{P}^{\eta}\left(l_{2}-l_{1}\right)}-\frac{\Gamma(\eta+1)}{l_{2}-l_{1}}\left[\left({ }^{\mathcal{P}} \mathcal{I}_{l_{1}^{+}}^{\eta} \hbar\right)(z)+\left({ }^{\mathcal{P}} \mathcal{I}_{l_{2}^{\eta}}^{\eta} \hbar\right)(z)\right]\right| \\
\leq \frac{\left(z^{\mathcal{P}}-l_{1}^{\mathcal{P}}\right)^{\eta+1}+\left(l_{2}^{\mathcal{P}}-z^{\mathcal{P}}\right)^{\eta+1}}{\mathcal{P}^{1+\eta}\left(l_{2}-l_{1}\right)}\left[\frac{\left(l_{2}^{\alpha(1-\mathcal{P})}\right)}{\alpha(\alpha \eta+1)}+\frac{1}{\beta}\left(\frac{\mathcal{M}}{n} \sum_{\theta=1}^{n} \frac{2 \theta}{\theta+1}\right)^{\beta}\right]
\end{gathered}
$$

holds for all $z \in\left(l_{1}, l_{2}\right)$ and $\mathcal{P} \in(-\infty, 0) \cup(0,1)$.

Proof We need and recall the Young inequality as follows:

$$
c d \leq \frac{1}{\alpha} c^{\alpha}+\frac{1}{\beta} d^{\alpha}, \quad c, d \geq 0, \alpha, \beta>1, \alpha^{-1}+\beta^{-1}=1 .
$$

To prove the first inequality of Theorem 5.5, we use Lemma 5.1, (5.5) and the $n$-polynomial $\mathcal{P}$-convexity of $\left|\hbar^{\prime}\right|^{\beta}$ to obtain

$$
\begin{aligned}
& \left|\frac{\left(z^{\mathcal{P}}-l_{1}^{\mathcal{P}}\right)^{\eta} \hbar\left(l_{1}\right)+\left(l_{2}^{\mathcal{P}}-z^{\mathcal{P}}\right)^{\eta} \hbar\left(l_{2}\right)}{\mathcal{P}^{\eta}\left(l_{2}-l_{1}\right)}-\frac{\Gamma(\eta+1)}{l_{2}-l_{1}}\left[\left({ }^{\mathcal{P}} \mathcal{I}_{l_{1}^{+}}^{\eta} \hbar\right)(z)+\left({ }^{\mathcal{P}} \mathcal{I}_{l_{2}}^{\eta} \hbar\right)(z)\right]\right| \\
& \leq \frac{\left(z^{\mathcal{P}}-l_{1}^{\mathcal{P}}\right)^{\eta+1}}{\mathcal{P}^{1+\eta}\left(l_{2}-l_{1}\right)} \\
& \quad \times \int_{0}^{1}\left(\frac{1}{\alpha}\left|\xi^{\eta}\left(\xi l_{1}^{\mathcal{P}}+(1-\xi) z^{\mathcal{P}}\right)^{\frac{1-\mathcal{P}}{\mathcal{P}}}\right|^{\alpha}+\frac{1}{\beta}\left|\hbar^{\prime}\left(\mathcal{P} \sqrt{\xi l_{1}^{\mathcal{P}}+(1-\xi) z^{\mathcal{P}}}\right)\right|^{\beta}\right) d \xi
\end{aligned}
$$




$$
\begin{aligned}
& +\frac{\left(l_{2}^{\mathcal{P}}-z^{\mathcal{P}}\right)^{\eta+1}}{\mathcal{P}^{1+\eta}\left(l_{2}-l_{1}\right)} \\
& \times \int_{0}^{1}\left(\frac{1}{\alpha}\left|\xi^{\eta}\left(\xi l_{2}^{\mathcal{P}}+(1-\xi) z^{\mathcal{P}}\right)^{\frac{1-\mathcal{P}}{\mathcal{P}}}\right|^{\alpha}+\frac{1}{\beta}\left|\hbar^{\prime}\left(\mathcal{P} \sqrt{\xi l_{2}^{\mathcal{P}}+(1-\xi) z^{\mathcal{P}}}\right)\right|^{\beta}\right) d \xi \\
\leq & \frac{\left(z^{\mathcal{P}}-l_{1}^{\mathcal{P}}\right)^{\eta+1}}{\mathcal{P}^{1+\eta}\left(l_{2}-l_{1}\right)}\left(\frac{\xi^{\alpha \eta}}{\alpha}\left|\left(\xi l_{1}^{\mathcal{P}}+(1-\xi) z^{\mathcal{P}}\right)^{\frac{1-\mathcal{P}}{\mathcal{P}}}\right|^{\alpha}\right. \\
& \left.+\frac{1}{\beta}\left|\frac{1}{n} \sum_{\theta=1}^{n}\left[1-(1-\xi)^{\theta}\right]\right| \hbar^{\prime}\left(l_{1}\right)\left|+\frac{1}{n} \sum_{\theta=1}^{n}\left[1-\xi^{\theta}\right]\right| \hbar^{\prime}(z)||^{\beta}\right) d \xi \\
& +\frac{\left(l_{2}^{\mathcal{P}}-z^{\mathcal{P}}\right)^{\eta+1}}{\mathcal{P}^{1+\eta}\left(l_{2}-l_{1}\right)} \int_{0}^{1}\left(\frac{\xi^{\alpha \eta}}{\alpha}\left|\left(\xi l_{2}^{\mathcal{P}}+(1-\xi) z^{\mathcal{P}}\right)^{\frac{1-\mathcal{P}}{\mathcal{P}}}\right|^{\alpha}\right. \\
& \left.+\frac{1}{\beta}\left|\frac{1}{n} \sum_{\theta=1}^{n}\left[1-(1-\xi)^{\theta}\right]\right| \hbar^{\prime}\left(l_{2}\right)\left|+\frac{1}{n} \sum_{\theta=1}^{n}\left[1-\xi^{\theta}\right]\right| \hbar^{\prime}(z)||^{\beta}\right) d \xi \\
\leq & \frac{\left(z^{\mathcal{P}}-l_{1}^{\mathcal{P}}\right)^{\eta+1}+\left(l_{2}^{\mathcal{P}}-z^{\mathcal{P}}\right)^{\eta+1}}{\mathcal{P}^{1+\eta}\left(l_{2}-l_{1}\right)}\left[\frac{\left(l_{1}^{\alpha(1-\mathcal{P})}\right)}{\alpha(\alpha \eta+1)}+\frac{1}{\beta}\left(\frac{\mathcal{M}}{n} \sum_{\theta=1}^{n} \frac{2 \theta}{\theta+1}\right)^{\beta}\right] .
\end{aligned}
$$

The second inequality of Theorem 5.5 can be proved in a similar way by the use of (5.6).

Theorem 5.6 Let $n \in \mathbb{N}, \alpha, \beta>1$ with $\alpha+\beta=1, l_{1}, l_{2} \in \Omega$ with $l_{1}<l_{2}$, and $\hbar: \Omega \subset(0, \infty) \rightarrow$ $\mathbb{R}$ be a differentiable function on $\Omega^{\circ}$ such that $\hbar^{\prime} \in L_{1}\left(\left[l_{1}, l_{2}\right]\right)$ and $\left|\hbar^{\prime}\right|^{\beta}$ is a n-polynomial $\mathcal{P}$-convex function satisfies $\left|\hbar^{\prime}(z)\right| \leq \mathcal{M}$ for all $z \in\left[l_{1}, l_{2}\right]$. Then the inequality

$$
\begin{aligned}
& \left|\frac{\left(z^{\mathcal{P}}-l_{1}^{\mathcal{P}}\right)^{\eta} \hbar\left(l_{1}\right)+\left(l_{2}^{\mathcal{P}}-z^{\mathcal{P}}\right)^{\eta} \hbar\left(l_{2}\right)}{\mathcal{P}^{\eta}\left(l_{2}-l_{1}\right)}-\frac{\Gamma(\eta+1)}{l_{2}-l_{1}}\left[\left({ }^{\mathcal{P}} \mathcal{I}_{l_{1}^{+}}^{\eta} \hbar\right)(z)+\left({ }^{\mathcal{P}} \mathcal{I}_{l_{2}^{-}}^{\eta} \hbar\right)(z)\right]\right| \\
& \quad \leq \frac{\left(z^{\mathcal{P}}-l_{1}^{\mathcal{P}}\right)^{\eta+1}+\left(l_{2}^{\mathcal{P}}-z^{\mathcal{P}}\right)^{\eta+1}}{\mathcal{P}^{1+\eta}\left(l_{2}-l_{1}\right)}\left[\frac{\alpha l_{1}^{1-\mathcal{P}}}{(\eta+1)}+\frac{\beta \mathcal{M}}{n} \sum_{\theta=1}^{n} \frac{2 \theta}{\theta+1}\right]
\end{aligned}
$$

holds for all $z \in\left(l_{1}, l_{2}\right)$ and $\mathcal{P} \in(1, \infty)$, and the inequality

$$
\begin{aligned}
& \left|\frac{\left(z^{\mathcal{P}}-l_{1}^{\mathcal{P}}\right)^{\eta} \hbar\left(l_{1}\right)+\left(l_{2}^{\mathcal{P}}-z^{\mathcal{P}}\right)^{\eta} \hbar\left(l_{2}\right)}{\mathcal{P}^{\eta}\left(l_{2}-l_{1}\right)}-\frac{\Gamma(\eta+1)}{l_{2}-l_{1}}\left[\left({ }^{\mathcal{P}} \mathcal{I}_{l_{1}^{+}}^{\eta} \hbar\right)(z)+\left({ }^{\mathcal{P}} \mathcal{I}_{l_{2}}^{\eta} \hbar\right)(z)\right]\right| \\
& \quad \leq \frac{\left(z^{\mathcal{P}}-l_{1}^{\mathcal{P}}\right)^{\eta+1}+\left(l_{2}^{\mathcal{P}}-z^{\mathcal{P}}\right)^{\eta+1}}{\mathcal{P}^{1+\eta}\left(l_{2}-l_{1}\right)}\left[\frac{\alpha l_{2}^{1-\mathcal{P}}}{(\eta+1)}+\frac{\beta \mathcal{M}}{n} \sum_{\theta=1}^{n} \frac{2 \theta}{\theta+1}\right]
\end{aligned}
$$

holds for all $z \in\left(l_{1}, l_{2}\right)$ and $\mathcal{P} \in(-\infty, 0) \cup(0,1)$.

Proof We first recall the weighted $\mathcal{A M}-\mathcal{G M}$ inequality

$$
c^{\alpha} d^{\beta} \leq \alpha c+\beta d, \quad c, d \geq 0, \alpha, \beta>0, \alpha+\beta=1 .
$$


To prove the first inequality of Theorem 5.6, we use Lemma 5.1, (5.5) and the $n$-polynomial $\mathcal{P}$-convexity of $\left|\hbar^{\prime}\right|^{\beta}$ to get

$$
\begin{aligned}
& \left|\frac{\left(z^{\mathcal{P}}-l_{1}^{\mathcal{P}}\right)^{\eta} \hbar\left(l_{1}\right)+\left(l_{2}^{\mathcal{P}}-z^{\mathcal{P}}\right)^{\eta} \hbar\left(l_{2}\right)}{\mathcal{P}^{\eta}\left(l_{2}-l_{1}\right)}-\frac{\Gamma(\eta+1)}{l_{2}-l_{1}}\left[\left({ }^{\mathcal{P}} \mathcal{I}_{l_{1}^{+}}^{\eta} \hbar\right)(z)+\left({ }^{\mathcal{P}} \mathcal{I}_{l_{2}^{-}}^{\eta} \hbar\right)(z)\right]\right| \\
& \leq \frac{\left(z^{\mathcal{P}}-l_{1}^{\mathcal{P}}\right)^{\eta+1}}{\mathcal{P}^{1+\eta}\left(l_{2}-l_{1}\right)} \int_{0}^{1}\left[\xi^{\eta}\left(\xi l_{1}^{\mathcal{P}}+(1-\xi) z^{\mathcal{P}}\right)^{\frac{1-\mathcal{P}}{\mathcal{P}}}\right]^{\alpha}\left[\left|\hbar^{\prime}\left(\mathcal{P} \sqrt{\xi l_{1}^{\mathcal{P}}+(1-\xi) z^{\mathcal{P}}}\right)\right|\right]^{\beta} d \xi \\
& \left.+\frac{\left(l_{2}^{\mathcal{P}}-z^{\mathcal{P}}\right)^{\eta+1}}{\mathcal{P}^{1+\eta}\left(l_{2}-l_{1}\right)} \int_{0}^{1} \xi^{\eta}\left(\xi l_{2}^{\mathcal{P}}+(1-\xi) z^{\mathcal{P}}\right)^{\frac{1-\mathcal{P}}{\mathcal{P}}}\right]^{\alpha}\left[\left|\hbar^{\prime}\left(\mathcal{P} \sqrt{\xi l_{2}^{\mathcal{P}}+(1-\xi) z^{\mathcal{P}}}\right)\right|\right]^{\beta} d \xi \\
& \leq \frac{\left(z^{\mathcal{P}}-l_{1}^{\mathcal{P}}\right)^{\eta+1}}{\mathcal{P}^{1+\eta}\left(l_{2}-l_{1}\right)} \\
& \times\left[\int_{0}^{1} \alpha \xi^{\eta}\left|\left(\xi l_{1}^{\mathcal{P}}+(1-\xi) z^{\mathcal{P}}\right)^{\frac{1-\mathcal{P}}{\mathcal{P}}}\right| d \xi+\int_{0}^{1} \beta\left|\hbar^{\prime}\left(\mathcal{P} \sqrt{\xi l_{1}^{\mathcal{P}}+(1-\xi) z^{\mathcal{P}}}\right)\right| d \xi\right] \\
& +\frac{\left(l_{2}^{\mathcal{P}}-z^{\mathcal{P}}\right)^{\eta+1}}{\mathcal{P}^{1+\eta}\left(l_{2}-l_{1}\right)} \\
& \times\left[\int_{0}^{1} \alpha \xi^{\eta}\left|\left(\xi l_{1}^{\mathcal{P}}+(1-\xi) z^{\mathcal{P}}\right)^{\frac{1-\mathcal{P}}{\mathcal{P}}}\right| d \xi+\int_{0}^{1} \beta\left|\hbar^{\prime}\left(\mathcal{P} \sqrt{\xi l_{1}^{\mathcal{P}}+(1-\xi) z^{\mathcal{P}}}\right)\right| d \xi\right] \\
& \leq \frac{\left(z^{\mathcal{P}}-l_{1}^{\mathcal{P}}\right)^{\eta+1}}{\mathcal{P}^{1+\eta}\left(l_{2}-l_{1}\right)} \\
& \times\left[\int_{0}^{1} l_{1}^{1-\mathcal{P}} \alpha \xi^{\eta} d \xi+\int_{0}^{1} \beta\left|\frac{1}{n} \sum_{\theta=1}^{n}\left[1-(1-\xi)^{\theta}\right]\right| \hbar^{\prime}\left(l_{1}\right)\left|+\frac{1}{n} \sum_{\theta=1}^{n}\left[1-\xi^{\theta}\right]\right| \hbar^{\prime}(z)|| d \xi\right] \\
& +\frac{\left(l_{2}^{\mathcal{P}}-z^{\mathcal{P}}\right)^{\eta+1}}{\mathcal{P}^{1+\eta}\left(l_{2}-l_{1}\right)} \\
& \times\left[\int_{0}^{1} l_{1}^{1-\mathcal{P}} \alpha \xi^{\eta} d \xi+\int_{0}^{1} \beta\left|\frac{1}{n} \sum_{\theta=1}^{n}\left[1-(1-\xi)^{\theta}\right]\right| \hbar^{\prime}\left(l_{2}\right)\left|+\frac{1}{n} \sum_{\theta=1}^{n}\left[1-\xi^{\theta}\right]\right| \hbar^{\prime}(z)|| d \xi\right] \\
& \leq \frac{\left(z^{\mathcal{P}}-l_{1}^{\mathcal{P}}\right)^{\eta+1}+\left(l_{2}^{\mathcal{P}}-z^{\mathcal{P}}\right)^{\eta+1}}{\mathcal{P}^{1+\eta}\left(l_{2}-l_{1}\right)}\left[\frac{\alpha l_{1}^{1-\mathcal{P}}}{(\eta+1)}+\frac{\beta \mathcal{M}}{n} \sum_{\theta=1}^{n} \frac{2 \theta}{\theta+1}\right] \text {. }
\end{aligned}
$$

The second inequality of Theorem 5.6 can be proved in a similar way by using Eq. (5.6).

Theorem 5.7 Let $n \in \mathbb{N}, \alpha, \beta>1$ with $\alpha^{-1}+\beta^{-1}=1, l_{1}, l_{2} \in \Omega$ with $l_{1}<l_{2}$, and $\hbar: \Omega \subset$ $(0, \infty) \rightarrow \mathbb{R}$ be a differentiable function on $\Omega^{\circ}$ such that $\hbar^{\prime} \in L_{1}\left(\left[l_{1}, l_{2}\right]\right)$ and $\left|\hbar^{\prime}\right|^{\alpha}$ is a $n$ polynomial $\mathcal{P}$-convex function satisfies $\left|\hbar^{\prime}(z)\right| \leq \mathcal{M}$ for all $z \in\left[l_{1}, l_{2}\right]$. Then the inequality

$$
\begin{aligned}
& \left|\frac{\left(z^{\mathcal{P}}-l_{1}^{\mathcal{P}}\right)^{\eta} \hbar\left(l_{1}\right)+\left(l_{2}^{\mathcal{P}}-z^{\mathcal{P}}\right)^{\eta} \hbar\left(l_{2}\right)}{\mathcal{P}^{\eta}\left(l_{2}-l_{1}\right)}-\frac{\Gamma(\eta+1)}{l_{2}-l_{1}}\left[\left({ }^{\mathcal{P}} \mathcal{I}_{l_{1}^{+}}^{\eta} \hbar\right)(z)+\left({ }^{\mathcal{P}} \mathcal{I}_{l_{2}}^{\eta} \hbar\right)(z)\right]\right| \\
& \leq \frac{\left(z^{\mathcal{P}}-l_{1}^{\mathcal{P}}\right)^{\eta+1}}{\mathcal{P}^{1+\eta}\left(l_{2}-l_{1}\right)}\left[\left(\Delta_{1}\left(l_{1}, z ; \mathcal{P}\right)\right)^{1 / \alpha}\left(\frac{\mathcal{M}^{\beta}}{n} \sum_{\theta=1}^{n} \frac{\theta}{\theta+1}\right)^{1 / \beta}\right. \\
& \left.\quad+\left(\Delta_{2}\left(l_{1}, z ; \mathcal{P}\right)\right)^{1 / \alpha}\left(\frac{\mathcal{M}^{\beta}}{n} \sum_{\theta=1}^{n} \frac{\theta^{2}+2 \theta-1}{(\theta+2)(\theta+1)}\right)^{1 / \beta}\right]
\end{aligned}
$$


holds for all $z \in\left(l_{1}, l_{2}\right)$ and $\mathcal{P} \in(1, \infty)$, and the inequality

$$
\begin{aligned}
& \left|\frac{\left(z^{\mathcal{P}}-l_{1}^{\mathcal{P}}\right)^{\eta} \hbar\left(l_{1}\right)+\left(l_{2}^{\mathcal{P}}-z^{\mathcal{P}}\right)^{\eta} \hbar\left(l_{2}\right)}{\mathcal{P}^{\eta}\left(l_{2}-l_{1}\right)}-\frac{\Gamma(\eta+1)}{l_{2}-l_{1}}\left[\left({ }^{\mathcal{P}} \mathcal{I}_{l_{1}^{+}}^{\eta} \hbar\right)(z)+\left({ }^{\mathcal{P}} \mathcal{I}_{l_{2}^{\eta}}^{\eta} \hbar\right)(z)\right]\right| \\
& \leq \frac{\left(l_{2}^{\mathcal{P}}-z^{\mathcal{P}}\right)^{\eta+1}}{\mathcal{P}^{1+\eta}\left(l_{2}-l_{1}\right)}\left[\left(\Delta_{3}\left(l_{2}, z ; \mathcal{P}\right)\right)^{1 / \alpha}\left(\frac{\mathcal{M}^{\beta}}{n} \sum_{\theta=1}^{n} \frac{\theta}{\theta+1}\right)^{1 / \beta}\right. \\
& \left.\quad+\left(\Delta_{4}\left(l_{2}, z ; \mathcal{P}\right)\right)^{1 / \alpha}\left(\frac{\mathcal{M}^{\beta}}{n} \sum_{\theta=1}^{n} \frac{\theta^{2}+2 \theta-1}{(\theta+2)(\theta+1)}\right)^{1 / \beta}\right]
\end{aligned}
$$

holds for all $z \in\left(l_{1}, l_{2}\right)$ and $\mathcal{P} \in(-\infty, 0) \cup(0,1)$, where

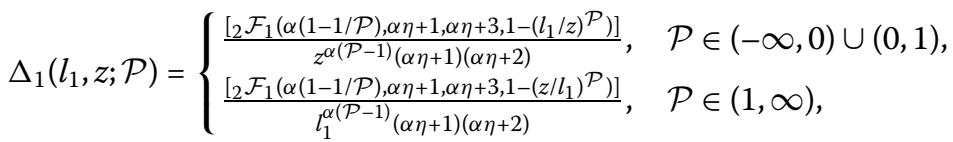

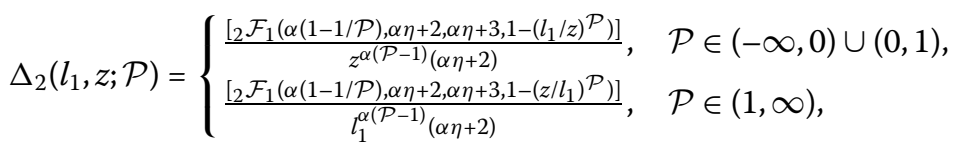

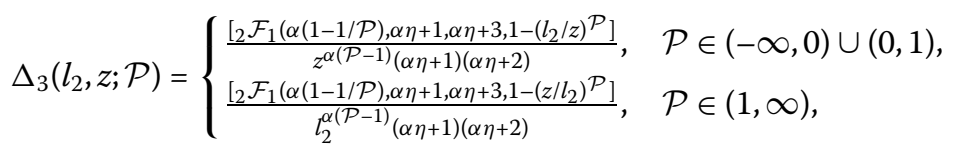

and

$$
\Delta_{4}\left(l_{2}, z ; \mathcal{P}\right)= \begin{cases}\frac{{ }_{2} \mathcal{F}_{1}\left(\alpha(1-1 / \mathcal{P}), \alpha \eta+2, \alpha \eta+3,1-\left(l_{2} / z\right)\right.}{\left.\left.z^{\alpha}\right)\right]}, & \mathcal{P} \in(-\infty, 0) \cup(0,1), \\ \frac{\left.{ }_{2} \mathcal{F}_{1}\left(\alpha(1-1 / \mathcal{P}),(\alpha \eta+2), \alpha \eta+3,1-\left(z / l_{2}\right)^{\mathcal{P}}\right)\right]}{l_{2}^{\alpha(\mathcal{P}-1)}(\alpha \eta+2)}, & \mathcal{P} \in(1, \infty) .\end{cases}
$$

Proof To prove the first inequality of Theorem 5.7, we use Lemma 5.1 and the Hölderİscan inequality to obtain

$$
\begin{aligned}
& \left|\frac{\left(z^{\mathcal{P}}-l_{1}^{\mathcal{P}}\right)^{\eta} \hbar\left(l_{1}\right)+\left(l_{2}^{\mathcal{P}}-z^{\mathcal{P}}\right)^{\eta} \hbar\left(l_{2}\right)}{\mathcal{P}^{\eta}\left(l_{2}-l_{1}\right)}-\frac{\Gamma(\eta+1)}{l_{2}-l_{1}}\left[\left({ }^{\mathcal{P}} \mathcal{I}_{l_{1}^{+}}^{\eta} \hbar\right)(z)+\left({ }^{\mathcal{P}} \mathcal{I}_{l_{2}^{-}}^{\eta} \hbar\right)(z)\right]\right| \\
& \leq \frac{\left(z^{\mathcal{P}}-l_{1}^{\mathcal{P}}\right)^{\eta+1}}{\mathcal{P}^{1+\eta}\left(l_{2}-l_{1}\right)} \int_{0}^{1} \xi^{\eta}\left(\xi l_{1}^{\mathcal{P}}+(1-\xi) z^{\mathcal{P}}\right)^{\frac{1-\mathcal{P}}{\mathcal{P}}}\left|\hbar^{\prime}\left(\mathcal{P} \sqrt{\xi l_{1}^{\mathcal{P}}+(1-\xi) z^{\mathcal{P}}}\right)\right| d \xi \\
& +\frac{\left(l_{2}^{\mathcal{P}}-z^{\mathcal{P}}\right)^{\eta+1}}{\mathcal{P}^{1+\eta}\left(l_{2}-l_{1}\right)} \int_{0}^{1} \xi^{\eta}\left(\xi l_{2}^{\mathcal{P}}+(1-\xi) z^{\mathcal{P}}\right)^{\frac{1-\mathcal{P}}{\mathcal{P}}}\left|\hbar^{\prime}\left({ }^{\mathcal{P}} \sqrt{\xi l_{2}^{\mathcal{P}}+(1-\xi) z^{\mathcal{P}}}\right)\right| d \xi \\
& \leq \frac{\left(z^{\mathcal{P}}-l_{1}^{\mathcal{P}}\right)^{\eta+1}}{\mathcal{P}^{1+\eta}\left(l_{2}-l_{1}\right)}\left[\left(\int_{0}^{1} \xi^{\alpha \eta}(1-\xi)\left(\xi l_{1}^{\mathcal{P}}+(1-\xi) z^{\mathcal{P}}\right)^{\alpha\left(\frac{1-\mathcal{P}}{\mathcal{P}}\right)} d \xi\right)^{1 / \alpha}\right. \\
& \times\left(\int_{0}^{1}(1-\xi)\left|\hbar^{\prime}\left(\mathcal{P}^{\mathcal{F}} \sqrt{\xi l_{1}^{\mathcal{P}}+(1-\xi) \mathcal{Z}^{\mathcal{P}}}\right)\right|^{\beta} d \xi\right)^{1 / \beta} \\
& +\left(\int_{0}^{1} \xi^{\alpha \eta+1}\left(\xi l_{1}^{\mathcal{P}}+(1-\xi) z^{\mathcal{P}}\right)^{\alpha\left(\frac{1-\mathcal{P}}{\mathcal{P}}\right)} d \xi\right)^{1 / \alpha} \\
& \left.\times\left(\int_{0}^{1} \xi\left|\hbar^{\prime}\left(\mathcal{P} \sqrt{\xi l_{1}^{\mathcal{P}}+(1-\xi) z^{\mathcal{P}}}\right)\right|^{\beta} d \xi\right)^{1 / \beta}\right]
\end{aligned}
$$


Chen et al. Advances in Difference Equations

(2020) 2020:543

Page 22 of 31

$$
\begin{aligned}
& +\frac{\left(l_{2}^{\mathcal{P}}-z^{\mathcal{P}}\right)^{\eta+1}}{\mathcal{P} 1+\eta\left(l_{2}-l_{1}\right)}\left[\left(\int_{0}^{1} \xi^{\alpha \eta}(1-\xi)\left(\xi l_{2}^{\mathcal{P}}+(1-\xi) z^{\mathcal{P}}\right)^{\alpha\left(\frac{1-\mathcal{P}}{\mathcal{P}}\right)} d \xi\right)^{1 / \alpha}\right. \\
& \times\left(\int_{0}^{1}(1-\xi)\left|\hbar^{\prime}\left(\mathcal{P} \sqrt{\xi l_{2}^{\mathcal{P}}+(1-\xi) z^{\mathcal{P}}}\right)\right|^{\beta} d \xi\right)^{1 / \beta} \\
& +\left(\int_{0}^{1} \xi^{\alpha \eta+1}\left(\xi l_{2}^{\mathcal{P}}+(1-\xi) z^{\mathcal{P}}\right)^{\alpha\left(\frac{1-\mathcal{P}}{\mathcal{P}}\right)} d \xi\right)^{1 / \alpha} \\
& \times\left(\int_{0}^{1} \xi \mid \hbar^{\prime}\left({ }^{\mathcal{P}} \sqrt{\left.\left.\left.\xi l_{2}^{\mathcal{P}}+(1-\xi) z^{\mathcal{P}}\right)\left.\right|^{\beta} d \xi\right)^{1 / \beta}\right]}\right.\right. \\
& \leq \frac{\left(z^{\mathcal{P}}-l_{1}^{\mathcal{P}}\right)^{\eta+1}}{\mathcal{P} 1+\eta\left(l_{2}-l_{1}\right)}\left[\left(\Delta_{1}\left(l_{1}, z ; \mathcal{P}\right)\right)^{1 / \alpha}\left(\int_{0}^{1}(1-\xi)\left|\hbar^{\prime}\left(\mathcal{P} \sqrt{\xi l_{1}^{\mathcal{P}}+(1-\xi) z^{\mathcal{P}}}\right)\right|^{\beta} d \xi\right)^{1 / \beta}\right. \\
& \left.+\left(\Delta_{2}\left(l_{1}, z ; \mathcal{P}\right)\right)^{1 / \alpha}\left(\int_{0}^{1} \xi\left|\hbar^{\prime}\left(\mathcal{P} \sqrt{\xi l_{1}^{\mathcal{P}}+(1-\xi) z^{\mathcal{P}}}\right)\right|^{\beta} d \xi\right)^{1 / \beta}\right] \\
& +\frac{\left(l_{2}^{\mathcal{P}}-z^{\mathcal{P}}\right)^{\eta+1}}{\mathcal{P}^{1+\eta}\left(l_{2}-l_{1}\right)}\left[\left(\Delta_{3}\left(l_{2}, z ; \mathcal{P}\right)\right)^{1 / \alpha}\left(\int_{0}^{1}(1-\xi)\left|\hbar^{\prime}\left(\mathcal{P}^{\mathcal{P}} \sqrt{\xi l_{2}^{\mathcal{P}}+(1-\xi) z^{\mathcal{P}}}\right)\right|^{\beta} d \xi\right)^{1 / \beta}\right. \\
& \left.+\left(\Delta_{4}\left(l_{2}, z ; \mathcal{P}\right)\right)^{1 / \alpha}\left(\int_{0}^{1} \xi\left|\hbar^{\prime}\left(\mathcal{P} \sqrt{\xi l_{2}^{\mathcal{P}}+(1-\xi) z^{\mathcal{P}}}\right)\right|^{\beta} d \xi\right)^{1 / \beta}\right] .
\end{aligned}
$$

Since $\left|\hbar^{\prime}\right|^{\beta}$ is $n$-polynomial $\mathcal{P}$-convex and $\left|\hbar^{\prime}(z)\right| \leq \mathcal{M}$ for all $z \in\left[l_{1}, l_{2}\right]$, we obtain

$$
\begin{aligned}
& \int_{0}^{1}(1-\xi)\left|\hbar^{\prime}\left(\mathcal{P} \sqrt{\xi l_{1}^{\mathcal{P}}+(1-\xi) z^{\mathcal{P}}}\right)\right|^{\beta} d \xi \\
& \quad \leq \int_{0}^{1}(1-\xi)\left[\frac{1}{n} \sum_{\theta=1}^{n}\left[1-(1-\xi)^{\theta}\right]\left|\hbar^{\prime}\left(l_{1}\right)\right|^{\beta}+\frac{1}{n} \sum_{\theta=1}^{n}\left[1-\xi^{\theta}\right]\left|\hbar^{\prime}(z)\right|^{\beta}\right] d \xi \\
& \quad \leq \frac{\mathcal{M}^{\beta}}{n} \sum_{\theta=1}^{n} \int_{0}^{1}\left[2(1-\xi)-(1-\xi)^{\theta+1}-\xi^{\theta}(1-\xi)\right] d \xi \\
& \quad=\frac{\mathcal{M}^{\beta}}{n} \sum_{\theta=1}^{n} \frac{\theta}{\theta+1} \\
& \int_{0}^{1} \xi\left|\hbar^{\prime}\left({ }^{\mathcal{P}} \sqrt{\xi l_{1}^{\mathcal{P}}+(1-\xi) z^{\mathcal{P}}}\right)\right|^{\beta} d \xi \\
& \leq \int_{0}^{1} \xi\left[\frac{1}{n} \sum_{\theta=1}^{n}\left[1-(1-\xi)^{\theta}\right]\left|\hbar^{\prime}\left(l_{1}\right)\right|^{\beta}+\frac{1}{n} \sum_{\theta=1}^{n}\left[1-\xi^{\theta}\right]\left|\hbar^{\prime}(z)\right|^{\beta}\right] d \xi \\
& \quad=\frac{\mathcal{M}^{\beta}}{n} \sum_{\theta=1}^{n} \int_{0}^{1}\left[2 \xi-\xi(1-\xi)^{\theta}-\xi^{\theta+1}\right] d \xi \\
& \leq \frac{\mathcal{M}^{\beta}}{n} \sum_{\theta=1}^{n} \frac{\theta^{2}+2 \theta-1}{(\theta+2)(\theta+1)} .
\end{aligned}
$$

Analogously, we have

$$
\int_{0}^{1}(1-\xi)\left|\hbar^{\prime}\left(\mathcal{P} \sqrt{\xi \mathcal{l}_{2}^{\mathcal{P}}+(1-\xi) z^{\mathcal{P}}}\right)\right|^{\beta} d \xi \leq \frac{\mathcal{M}^{\beta}}{n} \sum_{\theta=1}^{n} \frac{\theta}{\theta+1},
$$




$$
\int_{0}^{1} \xi\left|\hbar^{\prime}\left(\mathcal{P} \sqrt{\xi l_{2}^{\mathcal{P}}+(1-\xi) z^{\mathcal{P}}}\right)\right|^{\beta} d \xi \leq \frac{\mathcal{M}^{\beta}}{n} \sum_{\theta=1}^{n} \frac{\theta^{2}+2 \theta-1}{(\theta+2)(\theta+1)}
$$

Note that

$$
\begin{aligned}
& \Delta_{1}\left(l_{1}, z ; \mathcal{P}\right):=\int_{0}^{1} \xi^{\alpha \eta}(1-\xi)\left(\xi l_{1}^{\mathcal{P}}+(1-\xi) z^{\mathcal{P}}\right)^{\alpha\left(\frac{1-\mathcal{P}}{\mathcal{P}}\right)} d \xi \\
& = \begin{cases}\frac{{ }_{2} \mathcal{F}_{1}\left(\alpha(1-1 / \mathcal{P}), \alpha \eta+1, \alpha \eta+3,1-\left(l_{1} / z\right)^{\mathcal{P}}\right]}{z^{\alpha(\mathcal{P}-1)}(\alpha \eta+1)(\alpha \eta+2)}, & \mathcal{P} \in(-\infty, 0) \cup(0,1), \\
\frac{{ }_{2} \mathcal{F}_{1}\left(\alpha(1-1 / \mathcal{P}), \alpha \eta+1, \alpha \eta+3,1-\left(z / l_{1}\right)^{\mathcal{P}}\right]}{l_{1}^{\alpha(\mathcal{P}-1)},(\alpha \eta+1)(\alpha \eta+2)}, & \mathcal{P} \in(1, \infty),\end{cases} \\
& \Delta_{2}\left(l_{1}, z ; \mathcal{P}\right):=\int_{0}^{1} \xi^{\alpha \eta+1}\left(\xi l_{1}^{\mathcal{P}}+(1-\xi) z^{\mathcal{P}}\right)^{\alpha\left(\frac{1-\mathcal{P}}{\mathcal{P}}\right)} d \xi \\
& = \begin{cases}\frac{\left.{ }_{2} \mathcal{F}_{1}\left(\alpha(1-1 / \mathcal{P}), \alpha \eta+2, \alpha \eta+3,1-\left(l_{1} / z\right)^{\mathcal{P}}\right)\right]}{z^{\alpha(\mathcal{P}-1)}(\alpha \eta+2)}, & \mathcal{P} \in(-\infty, 0) \cup(0,1), \\
\frac{\left.{ }_{2} \mathcal{F}_{1}\left(\alpha(1-1 / \mathcal{P}), \alpha \eta+2, \alpha \eta+3,1-\left(z / l_{1}\right)^{\mathcal{P}}\right)\right]}{l_{1}^{\alpha(\mathcal{P}-1)}(\alpha \eta+2)}, & \mathcal{P} \in(1, \infty),\end{cases} \\
& \Delta_{3}\left(l_{2}, z ; \mathcal{P}\right):=\int_{0}^{1} \xi^{\alpha \eta}(1-\xi)\left(\xi l_{2}^{\mathcal{P}}+(1-\xi) z^{\mathcal{P}}\right)^{\alpha\left(\frac{1-\mathcal{P}}{\mathcal{P}}\right)} d \xi \\
& = \begin{cases}\frac{{ }_{2} \mathcal{F}_{1}\left(\alpha(1-1 / \mathcal{P}), \alpha \eta+1, \alpha \eta+3,1-\left(l_{2} / z\right)^{\mathcal{P}}\right]}{z^{\alpha(\mathcal{P}-1)}(\alpha \eta+1)(\alpha \eta+2)}, & \mathcal{P} \in(-\infty, 0) \cup(0,1), \\
\frac{{ }_{2} \mathcal{F}_{1}\left(\alpha(1-1 / \mathcal{P}), \alpha \eta+1, \alpha \eta+3,1-\left(z / l_{2}\right)^{\mathcal{P}}\right]}{l_{2}^{\alpha(\mathcal{P}-1)},(\alpha \eta+1)(\alpha \eta+2)}, & \mathcal{P} \in(1, \infty),\end{cases}
\end{aligned}
$$

and

$$
\begin{aligned}
\Delta_{4}\left(l_{2}, z ; \mathcal{P}\right): & \int_{0}^{1} \xi^{\alpha \eta+1}\left(\xi l_{2}^{\mathcal{P}}+(1-\xi) z^{\mathcal{P}}\right)^{\alpha\left(\frac{1-\mathcal{P}}{\mathcal{P}}\right)} d \xi \\
& = \begin{cases}\frac{{ }_{2} \mathcal{F}_{1}\left(\alpha(1-1 / \mathcal{P}), \alpha \eta+2, \alpha \eta+3,1-\left(l_{2} / z\right)\right.}{\left.\left.z^{\alpha}\right)\right]}, & \mathcal{P} \in(-\infty, 0) \cup(0,1), \\
\frac{\left[{ }_{2} \mathcal{F}_{1}\left(\alpha(1-1 / \mathcal{P}), \alpha \eta+2, \alpha \eta+3,1-\left(z / l_{2}\right)^{\mathcal{P}}\right)\right]}{l_{2}^{\alpha(\mathcal{P}-1)}(\alpha \eta+2)}, & \mathcal{P} \in(1, \infty) .\end{cases}
\end{aligned}
$$

Theorem 5.8 Let $n \in \mathbb{N}, \alpha, \beta>1$ with $\alpha^{-1}+\beta^{-1}=1, l_{1}, l_{2} \in \Omega$ with $l_{1}<l_{2}$, and $\hbar: \Omega \subset$ $(0, \infty) \rightarrow \mathbb{R}$ be a differentiable function on $\Omega^{\circ}$ such that $\hbar^{\prime} \in L_{1}\left(\left[l_{1}, l_{2}\right]\right)$ and $\left|\hbar^{\prime}\right|^{\alpha}$ is a $n$ polynomial convex function satisfies $\left|\hbar^{\prime}(z)\right| \leq \mathcal{M}$ for all $z \in\left[l_{1}, l_{2}\right]$. Then the inequality

$$
\begin{aligned}
& \left|\frac{\left(z^{\mathcal{P}}-l_{1}^{\mathcal{P}}\right)^{\eta} \hbar\left(l_{1}\right)+\left(l_{2}^{\mathcal{P}}-z^{\mathcal{P}}\right)^{\eta} \hbar\left(l_{2}\right)}{\mathcal{P}^{\eta}\left(l_{2}-l_{1}\right)}-\frac{\Gamma(\eta+1)}{l_{2}-l_{1}}\left[\left({ }^{\mathcal{P}} \mathcal{I}_{l_{1}^{+}}^{\eta} \hbar\right)(z)+\left({ }^{\mathcal{P}} \mathcal{I}_{l_{2}^{-}}^{\eta} \hbar\right)(z)\right]\right| \\
& \leq \frac{\left(z^{\mathcal{P}}-l_{1}^{\mathcal{P}}\right)^{\eta+1}}{\mathcal{P}^{1+\eta}\left(l_{2}-l_{1}\right)}\left[\left(\Delta_{1}^{*}\left(l_{1}, z ; \mathcal{P}\right)\right)^{1 / \alpha}\left(\frac{\mathcal{M}^{\beta}}{n} \sum_{\theta=1}^{n} \Upsilon_{1}\left(l_{1}, z ; \mathcal{P}\right)\right)^{1 / \beta}\right. \\
& \left.\quad+\left(\Delta_{2}^{*}\left(l_{1}, z ; \mathcal{P}\right)\right)^{1 / \alpha}\left(\frac{\mathcal{M}^{\beta}}{n} \sum_{\theta=1}^{n} \Upsilon_{2}\left(l_{1}, z ; \mathcal{P}\right)\right)^{1 / \beta}\right]
\end{aligned}
$$

holds for all $z \in\left(l_{1}, l_{2}\right)$ and $\mathcal{P} \in(1, \infty)$, and the inequality

$$
\left|\frac{\left(z^{\mathcal{P}}-l_{1}^{\mathcal{P}}\right)^{\eta} \hbar\left(l_{1}\right)+\left(l_{2}^{\mathcal{P}}-z^{\mathcal{P}}\right)^{\eta} \hbar\left(l_{2}\right)}{\mathcal{P}^{\eta}\left(l_{2}-l_{1}\right)}-\frac{\Gamma(\eta+1)}{l_{2}-l_{1}}\left[\left({ }^{\mathcal{P}} \mathcal{I}_{l_{1}^{+}}^{\eta} \hbar\right)(z)+\left({ }^{\mathcal{P}} \mathcal{I}_{l_{2}^{-}}^{\eta} \hbar\right)(z)\right]\right|
$$




$$
\begin{aligned}
& \leq \frac{\left(l_{2}^{\mathcal{P}}-z^{\mathcal{P}}\right)^{\eta+1}}{\mathcal{P}^{1+\eta}\left(l_{2}-l_{1}\right)}\left[\left(\Delta_{3}^{*}\left(l_{2}, z ; \mathcal{P}\right)\right)^{1 / \alpha}\left(\frac{\mathcal{M}^{\beta}}{n} \sum_{\theta=1}^{n} \Upsilon_{3}\left(l_{2}, z ; \mathcal{P}\right)\right)^{1 / \beta}\right. \\
& \left.\quad+\left(\Delta_{4}^{*}\left(l_{2}, z ; \mathcal{P}\right)\right)^{1 / \alpha}\left(\frac{\mathcal{M}^{\beta}}{n} \sum_{\theta=1}^{n} \Upsilon_{4}\left(l_{2}, z ; \mathcal{P}\right)\right)^{1 / \beta}\right]
\end{aligned}
$$

holds for all $z \in\left(l_{1}, l_{2}\right)$ and $\mathcal{P} \in(-\infty, 0) \cup(0,1)$, where

$$
\begin{aligned}
& \Upsilon_{1}\left(l_{1} z ; \mathcal{P}\right)=\left\{\begin{aligned}
\frac{1}{z^{(\mathcal{P}-1)}}\left[\frac{2}{(\eta+1)(\eta+2)}{ }_{2} \mathcal{F}_{1}\left(1-1 / \mathcal{P}, \eta+1, \eta+3,1-\left(l_{1} / z\right)^{\mathcal{P}}\right)\right. \\
\left.\quad-\mathbb{B}(\eta+1, \theta+2)_{2} \mathcal{F}_{1}\left(1-1 / \mathcal{P}, \eta+1, \theta+\eta+3,1-\left(l_{1} / z\right)^{\mathcal{P}}\right)\right] \\
\left.\quad-\mathbb{B}(\eta+\theta+1,2)_{2} \mathcal{F}_{1}\left(1-1 / \mathcal{P}, \eta+\theta+1, \theta+\eta+3,1-\left(l_{1} / z\right)^{\mathcal{P}}\right)\right], \\
\mathcal{P} \in(-\infty, 0) \cup(0,1), \\
\frac{1}{l_{1}^{(\mathcal{P}-1)}}\left[\frac{2}{(\eta+1)(\eta+2)}{ }_{2} \mathcal{F}_{1}\left(1-1 / \mathcal{P}, \eta+1, \eta+3,1-\left(z / l_{1}\right)^{\mathcal{P}}\right)\right. \\
\left.-\mathbb{B}(\eta+1, \theta+2)_{2} \mathcal{F}_{1}\left(1-1 / \mathcal{P}, \eta+1, \theta+\eta+3,1-\left(z / l_{1}\right)^{\mathcal{P}}\right)\right] \\
\left.-\mathbb{B}(\eta+\theta+1,2)_{2} \mathcal{F}_{1}\left(1-1 / \mathcal{P}, \eta+\theta+1, \theta+\eta+3,1-\left(z / l_{1}\right)^{\mathcal{P}}\right)\right], \\
\mathcal{P} \in(1, \infty),
\end{aligned}\right. \\
& \left\{\begin{array}{l}
\frac{1}{z^{(\mathcal{P}-1)}}\left[\frac{2}{(\eta+2)}{ }_{2} \mathcal{F}_{1}\left(1-1 / \mathcal{P}, \eta+2, \eta+3,1-\left(l_{1} / z\right)^{\mathcal{P}}\right)\right. \\
\left.\quad-\mathbb{B}(\eta+2, \theta+2)_{2} \mathcal{F}_{1}\left(1-1 / \mathcal{P}, \eta+2, \theta+\eta+3,1-\left(l_{1} / z\right)^{\mathcal{P}}\right)\right]
\end{array}\right. \\
& \left.-\frac{1}{\eta+\theta+2} 2 \mathcal{F}_{1}\left(1-1 / \mathcal{P}, \eta+\theta+2, \theta+\eta+3,1-\left(l_{1} / z\right)^{\mathcal{P}}\right)\right] \text {, } \\
& \Upsilon_{2}\left(l_{1} z ; \mathcal{P}\right)=\left\{\begin{array}{l}
\mathcal{P} \in(-\infty, 0) \cup(0,1), \\
\frac{1}{l_{1}^{(\mathcal{P}-1)}}\left[\frac{2}{(\eta+2)}{ }_{2} \mathcal{F}_{1}\left(1-1 / \mathcal{P}, \eta+2, \eta+3,1-\left(z / l_{1}\right)^{\mathcal{P}}\right)\right.
\end{array}\right. \\
& \left.-\mathbb{B}(\eta+2, \theta+2)_{2} \mathcal{F}_{1}\left(1-1 / \mathcal{P}, \eta+2, \theta+\eta+3,1-\left(z / l_{1}\right)^{\mathcal{P}}\right)\right] \\
& \left.-\frac{1}{\eta+\theta+2} 2 \mathcal{F}_{1}\left(1-1 / \mathcal{P}, \eta+\theta+2, \theta+\eta+3,1-\left(z / l_{1}\right)^{\mathcal{P}}\right)\right] \text {, } \\
& \mathcal{P} \in(1, \infty) \text {, } \\
& \Delta_{1}^{*}\left(l_{1}, z ; \mathcal{P}\right)=\left\{\begin{array}{c}
\frac{1}{z^{\mathcal{P}-1}(\eta+1)(\eta+2)}{ }_{2} \mathcal{F}_{1}\left((1-1 / \mathcal{P}), \eta+1, \eta+3,1-\left(l_{1} / z\right)^{\mathcal{P}}\right), \\
\mathcal{P} \in(-\infty, 0) \cup(0,1), \\
\frac{1}{l_{1}^{\mathcal{P}-1}(\eta+1)(\eta+2)}{ }_{2} \mathcal{F}_{1}\left((1-1 / \mathcal{P}), \eta+1, \eta+3,1-\left(z / l_{1}\right)^{\mathcal{P}}\right), \\
\mathcal{P} \in(1, \infty),
\end{array}\right. \\
& \Delta_{2}^{*}\left(l_{1}, z ; \mathcal{P}\right)=\left\{\begin{array}{l}
\frac{1}{z^{\mathcal{P}-1}(\eta+2)}\left[{ }_{2} \mathcal{F}_{1}\left((1-1 / \mathcal{P}), \eta+2, a \eta+3,1-\left(l_{1} / z\right)^{\mathcal{P}}\right)\right] \\
\mathcal{P} \in(-\infty, 0) \cup(0,1), \\
\frac{1}{l_{1}^{\mathcal{P}-1}(\eta+2)}\left[{ }_{2} \mathcal{F}_{1}\left((1-1 / \mathcal{P}), \eta+2, a \eta+3,1-\left(z / l_{1}\right)^{\mathcal{P}}\right)\right] \\
\mathcal{P} \in(1, \infty)
\end{array}\right. \\
& \Delta_{3}^{*}\left(l_{2}, z ; \mathcal{P}\right)=\left\{\begin{array}{c}
\frac{1}{z^{\mathcal{P}-1}(\eta+1)(\eta+2)}{ }_{2} \mathcal{F}_{1}\left((1-1 / \mathcal{P}), \eta+1, \eta+3,1-\left(l_{2} / z\right)^{\mathcal{P}}\right), \\
\mathcal{P} \in(-\infty, 0) \cup(0,1), \\
\frac{1}{l_{2}^{\mathcal{P}-1}(\eta+1)(\eta+2)}{ }_{2} \mathcal{F}_{1}\left((1-1 / \mathcal{P}), \eta+1, \eta+3,1-\left(z / l_{2}\right)^{\mathcal{P}}\right), \\
\mathcal{P} \in(1, \infty),
\end{array}\right.
\end{aligned}
$$


and

$$
\Delta_{4}^{*}\left(l_{2}, z ; \mathcal{P}\right)=\left\{\begin{array}{l}
\frac{1}{z^{\mathcal{P}-1}(\eta+2)}\left[{ }_{2} \mathcal{F}_{1}\left((1-1 / \mathcal{P}), \eta+2, a \eta+3,1-\left(l_{2} / z\right)^{\mathcal{P}}\right)\right], \\
\mathcal{P} \in(-\infty, 0) \cup(0,1), \\
\frac{1}{\bar{l}_{2}^{\mathcal{P}-1}(\eta+2)}\left[{ }_{2} \mathcal{F}_{1}\left((1-1 / \mathcal{P}), \eta+2, a \eta+3,1-\left(z / l_{2}\right)^{\mathcal{P}}\right)\right], \\
\mathcal{P} \in(1, \infty) .
\end{array}\right.
$$

Proof It follows from Lemma 5.1 and the improved power-mean inequality that

$$
\begin{aligned}
& \left|\frac{\left(z^{\mathcal{P}}-l_{1}^{\mathcal{P}}\right)^{\eta} \hbar\left(l_{1}\right)+\left(l_{2}^{\mathcal{P}}-z^{\mathcal{P}}\right)^{\eta} \hbar\left(l_{2}\right)}{\mathcal{P}^{\eta}\left(l_{2}-l_{1}\right)}-\frac{\Gamma(\eta+1)}{l_{2}-l_{1}}\left[\left({ }^{\mathcal{P}} \mathcal{I}_{l_{1}^{+}}^{\eta} \hbar\right)(z)+\left({ }^{\mathcal{P}} \mathcal{I}_{l_{2}^{-}}^{\eta} \hbar\right)(z)\right]\right| \\
& \leq \frac{\left(z^{\mathcal{P}}-l_{1}^{\mathcal{P}}\right)^{\eta+1}}{\mathcal{P}^{1+\eta}\left(l_{2}-l_{1}\right)} \int_{0}^{1} \xi^{\eta}\left(\xi l_{1}^{\mathcal{P}}+(1-\xi) z^{\mathcal{P}}\right)^{\frac{1-\mathcal{P}}{\mathcal{P}}}\left|\hbar^{\prime}\left(\mathcal{P} \sqrt{\xi l_{1}^{\mathcal{P}}+(1-\xi) z^{\mathcal{P}}}\right)\right| d \xi \\
& +\frac{\left(l_{2}^{\mathcal{P}}-z^{\mathcal{P}}\right)^{\eta+1}}{\mathcal{P}^{1+\eta}\left(l_{2}-l_{1}\right)} \int_{0}^{1} \xi^{\eta}\left(\xi l_{2}^{\mathcal{P}}+(1-\xi) z^{\mathcal{P}}\right)^{\frac{1-\mathcal{P}}{\mathcal{P}}}\left|\hbar^{\prime}\left(\mathcal{P} \sqrt{\xi l_{2}^{\mathcal{P}}+(1-\xi) z^{\mathcal{P}}}\right)\right| d \xi \\
& \leq \frac{\left(z^{\mathcal{P}}-l_{1}^{\mathcal{P}}\right)^{\eta+1}}{\mathcal{P}^{1+\eta}\left(l_{2}-l_{1}\right)}\left[\left(\int_{0}^{1} \xi^{\eta}(1-\xi)\left(\xi l_{1}^{\mathcal{P}}+(1-\xi) z^{\mathcal{P}}\right)^{\left(\frac{1-\mathcal{P}}{\mathcal{P}}\right)} d \xi\right)^{1-1 / \alpha}\right. \\
& \times\left(\int_{0}^{1} \xi^{\eta}(1-\xi)\left(\xi l_{1}^{\mathcal{P}}+(1-\xi) z^{\mathcal{P}}\right)^{\left(\frac{1-\mathcal{P}}{\mathcal{P}}\right)}\left|\hbar^{\prime}\left(\mathcal{P} \sqrt{\xi l_{1}^{\mathcal{P}}+(1-\xi) z^{\mathcal{P}}}\right)\right|^{\beta} d \xi\right)^{1 / \beta} \\
& +\left(\int_{0}^{1} \xi^{\eta+1}\left(\xi l_{1}^{\mathcal{P}}+(1-\xi) z^{\mathcal{P}}\right)^{\left(\frac{1-\mathcal{P}}{\mathcal{P}}\right)} d \xi\right)^{1-1 / \alpha} \\
& \left.\times\left(\int_{0}^{1} \xi^{\eta+1}\left(\xi l_{1}^{\mathcal{P}}+(1-\xi) z^{\mathcal{P}}\right)^{\left(\frac{1-\mathcal{P}}{\mathcal{P}}\right)}\left|\hbar^{\prime}\left(\mathcal{P} \sqrt{\xi l_{1}^{\mathcal{P}}+(1-\xi) z^{\mathcal{P}}}\right)\right|^{\beta} d \xi\right)^{1 / \beta}\right] \\
& +\frac{\left(l_{2}^{\mathcal{P}}-z^{\mathcal{P}}\right)^{\eta+1}}{\mathcal{P}^{1+\eta}\left(l_{2}-l_{1}\right)}\left[\left(\int_{0}^{1} \xi^{\eta}(1-\xi)\left(\xi l_{2}^{\mathcal{P}}+(1-\xi) z^{\mathcal{P}}\right)^{\left(\frac{1-\mathcal{P}}{\mathcal{P}}\right)} d \xi\right)^{1-1 / \alpha}\right. \\
& \times\left(\int_{0}^{1} \xi^{\eta}(1-\xi)\left(\xi l_{2}^{\mathcal{P}}+(1-\xi) z^{\mathcal{P}}\right)^{\left(\frac{1-\mathcal{P}}{\mathcal{P}}\right)}\left|\hbar^{\prime}\left(\mathcal{P} \sqrt{\xi l_{2}^{\mathcal{P}}+(1-\xi) z^{\mathcal{P}}}\right)\right|^{\beta} d \xi\right)^{1 / \beta} \\
& +\left(\int_{0}^{1} \xi^{\eta+1}\left(\xi l_{2}^{\mathcal{P}}+(1-\xi) z^{\mathcal{P}}\right)^{\alpha\left(\frac{1-\mathcal{P}}{\mathcal{P}}\right)} d \xi\right)^{1-1 / \alpha} \\
& \left.\times\left(\int_{0}^{1} \xi^{\eta+1}\left(\xi l_{2}^{\mathcal{P}}+(1-\xi) z^{\mathcal{P}}\right)^{\alpha\left(\frac{1-\mathcal{P}}{\mathcal{P}}\right)}\left|\hbar^{\prime}\left(\mathcal{P}^{\mathcal{P}} \sqrt{\xi l_{2}^{\mathcal{P}}+(1-\xi) z^{\mathcal{P}}}\right)\right|^{\beta} d \xi\right)^{1 / \beta}\right] \\
& \leq \frac{\left(z^{\mathcal{P}}-l_{1}^{\mathcal{P}}\right)^{\eta+1}}{\mathcal{P}^{1+\eta}\left(l_{2}-l_{1}\right)}\left[\left(\Delta_{1}^{*}\left(l_{1}, z ; \mathcal{P}\right)\right)^{1-1 / \alpha}\right. \\
& \times\left(\int_{0}^{1} \xi^{\eta}(1-\xi)\left(\xi l_{1}^{\mathcal{P}}+(1-\xi) z^{\mathcal{P}}\right)^{\left(\frac{1-\mathcal{P}}{\mathcal{P}}\right)}\left|\hbar^{\prime}\left(\mathcal{P} \sqrt{\xi l_{1}^{\mathcal{P}}+(1-\xi) z^{\mathcal{P}}}\right)\right|^{\beta} d \xi\right)^{1 / \beta} \\
& +\left(\Delta_{2}^{*}\left(l_{1}, z ; \mathcal{P}\right)\right)^{1-1 / \alpha} \\
& \left.\times\left(\int_{0}^{1} \xi^{\eta+1}\left(\xi l_{1}^{\mathcal{P}}+(1-\xi) z^{\mathcal{P}}\right)^{\left(\frac{1-\mathcal{P}}{\mathcal{P}}\right)}\left|\hbar^{\prime}\left({ }^{\mathcal{P}} \sqrt{\xi l_{1}^{\mathcal{P}}+(1-\xi) z^{\mathcal{P}}}\right)\right|^{\beta} d \xi\right)^{1 / \beta}\right] \\
& +\frac{\left(l_{2}^{\mathcal{P}}-z^{\mathcal{P}}\right)^{\eta+1}}{\mathcal{P}^{1+\eta}\left(l_{2}-l_{1}\right)}\left[\left(\Delta_{3}^{*}\left(l_{2}, z ; \mathcal{P}\right)\right)^{1-1 / \alpha}\right.
\end{aligned}
$$




$$
\begin{aligned}
& \times\left(\int_{0}^{1} \xi^{\eta}(1-\xi)\left(\xi l_{2}^{\mathcal{P}}+(1-\xi) z^{\mathcal{P}}\right)^{\left(\frac{1-\mathcal{P}}{\mathcal{P}}\right)}\left|\hbar^{\prime}\left(\mathcal{P} \sqrt{\xi l_{2}^{\mathcal{P}}+(1-\xi) z^{\mathcal{P}}}\right)\right|^{\beta} d \xi\right)^{1 / \beta} \\
& +\left(\Delta_{4}^{*}\left(l_{2}, z ; \mathcal{P}\right)\right)^{1-1 / \alpha} \\
& \left.\times\left(\int_{0}^{1} \xi^{\eta+1}\left(\xi l_{2}^{\mathcal{P}}+(1-\xi) z^{\mathcal{P}}\right)^{\alpha\left(\frac{1-\mathcal{P}}{\mathcal{P}}\right)}\left|\hbar^{\prime}\left(\mathcal{P} \sqrt{\xi l_{2}^{\mathcal{P}}+(1-\xi) z^{\mathcal{P}}}\right)\right|^{\beta} d \xi\right)^{1 / \beta}\right] .
\end{aligned}
$$

Since $\left|\hbar^{\prime}\right|^{\beta}$ is $n$-polynomial $\mathcal{P}$-convex and $\left|\hbar^{\prime}(z)\right| \leq \mathcal{M}$ for all $z \in\left[l_{1}, l_{2}\right]$, we get

$$
\begin{aligned}
& \int_{0}^{1} \xi^{\eta}(1-\xi)\left(\xi l_{1}^{\mathcal{P}}+(1-\xi) z^{\mathcal{P}}\right)^{\left(\frac{1-\mathcal{P}}{\mathcal{P}}\right)}\left|\hbar^{\prime}\left(\mathcal{P} \sqrt{\xi l_{1}^{\mathcal{P}}+(1-\xi) z^{\mathcal{P}}}\right)\right|^{\beta} d \xi \\
& \leq \int_{0}^{1} \xi^{\eta}(1-\xi)\left(\xi l_{1}^{\mathcal{P}}+(1-\xi) z^{\mathcal{P}}\right)^{\left(\frac{1-\mathcal{P}}{\mathcal{P}}\right)} \\
& \quad \times\left[\frac{1}{n} \sum_{\theta=1}^{n}\left[1-(1-\xi)^{\theta}\right]\left|\hbar^{\prime}\left(l_{1}\right)\right|^{\beta}+\frac{1}{n} \sum_{\theta=1}^{n}\left[1-\xi^{\theta}\right]\left|\hbar^{\prime}(z)\right|^{\beta}\right] d \xi \\
&=\frac{\mathcal{M}^{\beta}}{n} \sum_{\theta=1}^{n} \int_{0}^{1}\left(\xi l_{1}^{\mathcal{P}}+(1-\xi) z^{\mathcal{P}}\right)^{\left(\frac{1-\mathcal{P}}{\mathcal{P}}\right)}\left[2 \xi^{\eta}(1-\xi)-\xi^{\eta}(1-\xi)^{\theta+1}-\xi^{\eta+\theta}(1-\xi)\right] d \xi \\
&=\frac{\mathcal{M}^{\beta}}{n} \sum_{\theta=1}^{n} \Upsilon_{1}\left(l_{1} z ; \mathcal{P}\right),
\end{aligned}
$$

where

$$
\begin{aligned}
\Upsilon_{1}\left(l_{1} z ; \mathcal{P}\right):= & \int_{0}^{1}\left(\xi l_{1}^{\mathcal{P}}+(1-\xi) z^{\mathcal{P}}\right)^{\left(\frac{1-\mathcal{P}}{\mathcal{P}}\right)} \\
& \times\left[2 \xi^{\eta}(1-\xi)-\xi^{\eta}(1-\xi)^{\theta+1}-\xi^{\eta+\theta}(1-\xi)\right] d \xi \\
= & \left\{\begin{array}{c}
\frac{1}{z^{(\mathcal{P}-1)}}\left[\frac{2}{(\eta+1)(\eta+2)}{ }^{2} \mathcal{F}_{1}\left(1-1 / \mathcal{P}, \eta+1, \eta+3,1-\left(l_{1} / z\right)^{\mathcal{P}}\right)\right. \\
\left.\quad-\mathbb{B}(\eta+1, \theta+2)_{2} \mathcal{F}_{1}\left(1-1 / \mathcal{P}, \eta+1, \theta+\eta+3,1-\left(l_{1} / z\right)^{\mathcal{P}}\right)\right] \\
\left.-\mathbb{B}(\eta+\theta+1,2)_{2} \mathcal{F}_{1}\left(1-1 / \mathcal{P}, \eta+\theta+1, \theta+\eta+3,1-\left(l_{1} / z\right)^{\mathcal{P}}\right)\right], \\
\mathcal{P} \in(-\infty, 0) \cup(0,1), \\
\frac{1}{l_{1}^{\mathcal{P}-1)}}\left[\frac{2}{(\eta+1)(\eta+2)}{ }^{2} \mathcal{F}_{1}\left(1-1 / \mathcal{P}, \eta+1, \eta+3,1-\left(z / l_{1}\right)^{\mathcal{P}}\right)\right. \\
\left.-\mathbb{B}(\eta+1, \theta+2)_{2} \mathcal{F}_{1}\left(1-1 / \mathcal{P}, \eta+1, \theta+\eta+3,1-\left(z / l_{1}\right)^{\mathcal{P}}\right)\right] \\
\left.-\mathbb{B}(\eta+\theta+1,2)_{2} \mathcal{F}_{1}\left(1-1 / \mathcal{P}, \eta+\theta+1, \theta+\eta+3,1-\left(z / l_{1}\right)^{\mathcal{P}}\right)\right], \\
\mathcal{P} \in(1, \infty) .
\end{array}\right.
\end{aligned}
$$

Analogously, we have

$$
\begin{aligned}
& \int_{0}^{1} \xi^{\eta+1}\left(\xi l_{1}^{\mathcal{P}}+(1-\xi) z^{\mathcal{P}}\right)^{\left(\frac{1-\mathcal{P}}{\mathcal{P}}\right)}\left|\hbar^{\prime}\left(\mathcal{P} \sqrt{\xi l_{1}^{\mathcal{P}}+(1-\xi) z^{\mathcal{P}}}\right)\right|^{\beta} d \xi \\
& \leq \int_{0}^{1} \xi^{\eta+1}\left(\xi l_{1}^{\mathcal{P}}+(1-\xi) z^{\mathcal{P}}\right)^{\left(\frac{1-\mathcal{P}}{\mathcal{P}}\right)} \\
& \quad \times\left[\frac{1}{n} \sum_{\theta=1}^{n}\left[1-(1-\xi)^{\theta}\right]\left|\hbar^{\prime}\left(l_{1}\right)\right|^{\beta}+\frac{1}{n} \sum_{\theta=1}^{n}\left[1-\xi^{\theta}\right]\left|\hbar^{\prime}(z)\right|^{\beta}\right] d \xi
\end{aligned}
$$




$$
\begin{aligned}
& =\frac{\mathcal{M}^{\beta}}{n} \sum_{\theta=1}^{n} \int_{0}^{1}\left(\xi l_{1}^{\mathcal{P}}+(1-\xi) z^{\mathcal{P}}\right)^{\left(\frac{1-\mathcal{P}}{\mathcal{P}}\right)}\left[2 \xi^{\eta+1}-\xi^{\eta+1}(1-\xi)^{\theta}-\xi^{\eta+\theta+1}\right] d \xi \\
& =\frac{\mathcal{M}^{\beta}}{n} \sum_{\theta=1}^{n} \Upsilon_{2}\left(l_{1} z ; \mathcal{P}\right)
\end{aligned}
$$

where

$$
\begin{aligned}
& \Upsilon_{2}\left(l_{1} z ; \mathcal{P}\right):=\int_{0}^{1}\left(\xi l_{1}^{\mathcal{P}}+(1-\xi) z^{\mathcal{P}}\right)^{\left(\frac{1-\mathcal{P}}{\mathcal{P}}\right)}\left[2 \xi^{\eta+1}-\xi^{\eta+1}(1-\xi)^{\theta}-\xi^{\eta+\theta+1}\right] d \xi \\
& =\left\{\begin{array}{l}
\frac{1}{z^{(\mathcal{P}-1)}}\left[\frac{2}{(\eta+2)}{ }^{2} \mathcal{F}_{1}\left(1-1 / \mathcal{P}, \eta+2, \eta+3,1-\left(l_{1} / z\right)^{\mathcal{P}}\right)\right. \\
\left.\quad-\mathbb{B}(\eta+2, \theta+2)_{2} \mathcal{F}_{1}\left(1-1 / \mathcal{P}, \eta+2, \theta+\eta+3,1-\left(l_{1} / z\right)^{\mathcal{P}}\right)\right] \\
\left.\quad-\frac{1}{\eta+\theta+2} 2 \mathcal{F}_{1}\left(1-1 / \mathcal{P}, \eta+\theta+2, \theta+\eta+3,1-\left(l_{1} / z\right)^{\mathcal{P}}\right)\right], \\
\\
\mathcal{P} \in(-\infty, 0) \cup(0,1), \\
\frac{1}{l_{1}^{(\mathcal{P}-1)}}\left[\frac{2}{(\eta+2)}{ }^{2} \mathcal{F}_{1}\left(1-1 / \mathcal{P}, \eta+2, \eta+3,1-\left(z / l_{1}\right)^{\mathcal{P}}\right)\right. \\
\left.\quad-\mathbb{B}(\eta+2, \theta+2)_{2} \mathcal{F}_{1}\left(1-1 / \mathcal{P}, \eta+2, \theta+\eta+3,1-\left(z / l_{1}\right)^{\mathcal{P}}\right)\right] \\
\left.\quad-\frac{1}{\eta+\theta+2} 2 \mathcal{F}_{1}\left(1-1 / \mathcal{P}, \eta+\theta+2, \theta+\eta+3,1-\left(z / l_{1}\right)^{\mathcal{P}}\right)\right], \\
\mathcal{P} \in(1, \infty) .
\end{array}\right.
\end{aligned}
$$

In a similar way, we can obtain $\Upsilon_{3}\left(l_{2}, z ; \mathcal{P}\right)$ and $\Upsilon_{4}\left(l_{2}, z ; \mathcal{P}\right)$ by replacing $l_{1}$ into $l_{2}$ in (5.25) and (5.27) and applying the facts that

$$
\begin{aligned}
& \Delta_{1}^{*}\left(l_{1}, z ; \mathcal{P}\right):=\int_{0}^{1} \xi^{\eta}(1-\xi)\left(\xi l_{1}^{\mathcal{P}}+(1-\xi) z^{\mathcal{P}}\right)^{\left(\frac{1-\mathcal{P}}{\mathcal{P}}\right)} d \xi \\
& =\left\{\begin{array}{l}
\frac{1}{z^{\mathcal{P}-1}(\eta+1)(\eta+2)}{ }^{2} \mathcal{F}_{1}\left((1-1 / \mathcal{P}), \eta+1, \eta+3,1-\left(l_{1} / z\right)^{\mathcal{P}}\right), \\
\mathcal{P} \in(-\infty, 0) \cup(0,1), \\
\frac{1}{l_{1}^{\mathcal{P}-1}(\eta+1)(\eta+2)}{ }^{2} \mathcal{F}_{1}\left((1-1 / \mathcal{P}), \eta+1, \eta+3,1-\left(z / l_{1}\right)^{\mathcal{P}}\right), \\
\mathcal{P} \in(1, \infty),
\end{array}\right. \\
& \Delta_{2}^{*}\left(l_{1}, z ; \mathcal{P}\right):=\int_{0}^{1} \xi^{\eta+1}\left(\xi l_{1}^{\mathcal{P}}+(1-\xi) z^{\mathcal{P}}\right)^{\left(\frac{1-\mathcal{P}}{\mathcal{P}}\right)} d \xi \\
& =\left\{\begin{array}{l}
\frac{1}{z^{\mathcal{P}-1}(\eta+2)}\left[\mathcal{F}_{1}\left((1-1 / \mathcal{P}), \eta+2, a \eta+3,1-\left(l_{1} / z\right)^{\mathcal{P}}\right)\right], \\
\mathcal{P} \in(-\infty, 0) \cup(0,1), \\
\frac{1}{l_{1}^{\mathcal{P}-1}(\eta+2)}\left[2 \mathcal{F}_{1}\left((1-1 / \mathcal{P}), \eta+2, a \eta+3,1-\left(z / l_{1}\right)^{\mathcal{P}}\right)\right], \\
\mathcal{P} \in(1, \infty),
\end{array}\right. \\
& \Delta_{3}^{*}\left(l_{2}, z ; \mathcal{P}\right):=\int_{0}^{1} \xi^{\eta}(1-\xi)\left(\xi l_{2}^{\mathcal{P}}+(1-\xi) z^{\mathcal{P}}\right)^{\left(\frac{1-\mathcal{P}}{\mathcal{P}}\right)} d \xi \\
& =\left\{\begin{array}{l}
\frac{1}{\bar{z}^{\mathcal{P}-1}(\eta+1)(\eta+2)}{ }^{2} \mathcal{F}_{1}\left((1-1 / \mathcal{P}), \eta+1, \eta+3,1-\left(l_{2} / z\right)^{\mathcal{P}}\right), \\
\mathcal{P} \in(-\infty, 0) \cup(0,1), \\
\frac{1}{l_{2}^{\mathcal{P}-1}(\eta+1)(\eta+2)}{ }_{2} \mathcal{F}_{1}\left((1-1 / \mathcal{P}), \eta+1, \eta+3,1-\left(z / l_{2}\right)^{\mathcal{P}}\right), \\
\mathcal{P} \in(1, \infty),
\end{array}\right.
\end{aligned}
$$


and

$$
\begin{aligned}
\Delta_{4}^{*}\left(l_{2}, z ; \mathcal{P}\right): & \int_{0}^{1} \xi^{\eta+1}\left(\xi l_{2}^{\mathcal{P}}+(1-\xi) z^{\mathcal{P}}\right)^{\frac{1-\mathcal{P}}{\mathcal{P}}} d \xi \\
& =\left\{\begin{array}{c}
\frac{1}{z^{\mathcal{P}-1(\eta+2)}}\left[{ }_{2} \mathcal{F}_{1}\left((1-1 / \mathcal{P}), \eta+2, a \eta+3,1-\left(l_{2} / z\right)^{\mathcal{P}}\right)\right] \\
\mathcal{P} \in(-\infty, 0) \cup(0,1), \\
\frac{1}{l_{2}^{\mathcal{P}-1}(\eta+2)}\left[{ }_{2} \mathcal{F}_{1}\left((1-1 / \mathcal{P}), \eta+2, a \eta+3,1-\left(z / l_{2}\right)^{\mathcal{P}}\right)\right] \\
\mathcal{P} \in(1, \infty) .
\end{array}\right.
\end{aligned}
$$

This completes the proof.

\section{Inequalities for special function}

\subsection{Modified Bessel function}

Recalling the series representation of the first kind modified Bessel function [38]

$$
\mathcal{U}_{\rho}(z)=\sum_{n \geq 0} \frac{(z / 2)^{\rho+2 n}}{n ! \Gamma(\rho+n+1)} \quad(z \in \mathbb{R})
$$

Analogously, the second kind modified Bessel function $\mathcal{K}_{\rho}$ is defined by

$$
\mathcal{K}_{\rho}(z)=\frac{\pi}{2} \frac{\mathcal{U}_{-\rho}(z)+\mathcal{U}_{\rho}(z)}{\sin \rho \pi}
$$

Let $\omega_{\rho}: \mathbb{R} \rightarrow[1, \infty)$ be defined by

$$
\omega_{\rho}(z)=2^{\rho} \Gamma(\rho+1) z^{-\rho} \mathcal{U}_{\rho}(z)
$$

Proposition 6.1 Let $\rho>-1$ and $l_{2}>l_{1}>0$. Then one has

$$
\begin{aligned}
& \left|\frac{1}{2}\left[\omega_{\rho}\left(l_{1}\right)+\omega_{\rho}\left(l_{2}\right)\right]-\frac{1}{l_{2}-l_{1}} \int_{l_{1}}^{l_{2}} \omega_{\rho}(z) d z\right| \\
& \leq \frac{l_{2}-l_{1}}{4(\rho+1)^{1 / \beta}}\left(\frac{1}{2}\right)^{1-3 / \beta}\left[\frac{1}{n} \sum_{\theta=1}^{n} \frac{\left(\theta^{2}+\theta+2\right) 2^{\theta}-2}{(\theta+1)(\theta+2) 2^{\theta+1}}\right]^{1 / \beta} \\
& \quad \times\left[l_{1}\left|\omega_{\rho+1}\left(l_{1}\right)\right|^{\beta}+l_{2}\left|\omega_{\rho+1}\left(l_{2}\right)\right|^{\beta}\right]^{1 / \beta}
\end{aligned}
$$

In particular, if $\rho=-1 / 2$, then

$$
\begin{aligned}
& \left|\frac{1}{2}\left[\cosh \left(l_{1}\right)+\cosh \left(l_{2}\right)\right]-\frac{1}{l_{2}-l_{1}} \int_{l_{1}}^{l_{2}} \cosh (z) d z\right| \\
& \leq \frac{l_{2}-l_{1}}{2}\left(\frac{1}{2}\right)^{1-3 / \beta}\left[\frac{1}{n} \sum_{\theta=1}^{n} \frac{\left(\theta^{2}+\theta+2\right) 2^{\theta}-2}{(\theta+1)(\theta+2) 2^{\theta+1}}\right]^{1 / \beta} \\
& \quad \times\left[\left|\sinh \left(l_{1}\right)\right|^{\beta}+\left|\sinh \left(l_{2}\right)\right|^{\beta}\right]^{1 / \beta} .
\end{aligned}
$$

Proof Let $\mathcal{P}=\eta=1$. Then the desired inequality (6.4) can be derived by applying inequality (4.2) to the mapping $\hbar(z)=\omega_{\rho}(z)$ with $\omega_{\rho}^{\prime}(z)=\frac{z}{\rho+1} \omega_{\rho+1}$. 
If $\rho=-1 / 2$, then inequality (6.4) reduces to (6.5) due to $\omega_{-1 / 2}(z)=\cosh z$ and $\omega_{1 / 2}(z)=$ $\frac{\sin h(z)}{z}$.

\section{$6.2 \mathfrak{q}$-Digamma function}

Let $\mathfrak{q} \in(0,1)$. Then the $\mathfrak{q}$-analogue of the digamma function $\check{\phi}_{\mathfrak{q}}$ is given by

$$
\begin{aligned}
\check{\phi}_{\mathfrak{q}}(z) & =-\ln (1-\mathfrak{q})+\ln \mathfrak{q} \sum_{\mathcal{K}=0}^{\infty} \frac{\mathfrak{q}^{\mathcal{K}+z}}{1-\mathfrak{q}^{\mathcal{K}+z}} \\
& =-\ln (1-\mathfrak{q})+\ln \mathfrak{q} \sum_{\mathcal{K}=0}^{\infty} \frac{\mathfrak{q}^{-\mathcal{K} z}}{1-\mathfrak{q}^{\mathcal{K}}} .
\end{aligned}
$$

If $\mathfrak{q}>1$ and $z>0$, then the $\mathfrak{q}$-digamma function $\check{\phi}_{\mathfrak{q}}$ can be expressed as

$$
\begin{aligned}
\check{\phi}_{\mathfrak{q}}(z) & =-\ln (1-\mathfrak{q})+\ln \mathfrak{q}\left[z-\frac{1}{2}-\sum_{\mathcal{K}=0}^{\infty} \frac{\mathfrak{q}^{-(\mathcal{K}+z)}}{1-\mathfrak{q}^{-(\mathcal{K}+z)}}\right] \\
& =-\ln (1-\mathfrak{q})+\ln \mathfrak{q}\left[z-\frac{1}{2}-\sum_{\mathcal{K}=0}^{\infty} \frac{\mathfrak{q}^{-\mathcal{K} z}}{1-\mathfrak{q}^{-\mathcal{K} z}}\right] .
\end{aligned}
$$

Proposition 6.2 Let $\mathfrak{q} \in(0,1)$ and $l_{2}>l_{1}>0$. Then the inequality

$$
\begin{aligned}
& \left|\frac{\check{\phi}_{\mathfrak{q}}{ }^{\prime}\left(l_{1}\right)+\check{\phi}_{\mathfrak{q}}{ }^{\prime}\left(l_{2}\right)}{2}-\left(\frac{\check{\phi_{\mathfrak{q}}}\left(l_{2}\right)-\check{\phi}_{\mathfrak{q}}{ }^{\prime}\left(l_{1}\right)}{l_{2}-l_{1}}\right)\right| \\
& \leq \frac{l_{2}-l_{1}}{2}\left(\frac{1}{2}\right)^{1-3 / \beta}\left[\frac{1}{n} \sum_{\theta=1}^{n} \frac{\left(\theta^{2}+\theta+2\right) 2^{\theta}-2}{(\theta+1)(\theta+2) 2^{\theta+1}}\right]^{1 / \beta} \\
& \quad \times\left[\left|\check{\phi}_{\mathfrak{q}}^{(2)}\left(l_{1}\right)\right|^{\beta}+\left|\check{\phi}_{\mathfrak{q}}^{(2)}\left(l_{2}\right)\right|^{\beta}\right]^{1 / \beta}
\end{aligned}
$$

holds for all $z>0$.

Proof Let $\eta=\mathcal{P}=1$. Then from the definition of $\check{\phi}_{\mathfrak{q}}$ we know that the function $z \rightarrow \check{\phi}_{\mathfrak{q}}{ }^{\prime}(z)$ is a completely monotone function and is convex on $(0, \infty)$. Therefore, inequality $(6.8)$ can be derived by Theorem 4.2 immediately.

\section{Conclusions}

A novel idea of $n$-polynomial $\mathcal{P}$-convex function with several types of convexities is elaborated. By considering two identities for the generalized fractional integral operators, we proposed several novel generalizations for $n$-polynomial $\mathcal{P}$-convex functions. Here, we emphasized that all computed outcomes in the present investigation endured preserving for $n$-polynomial harmonically convex, $n$-polynomial convex, classical harmonically convex and classical convex functions that can be obtained by choosing $\mathcal{P}=-1$ or 1 and $\theta=1$. So the suggested technique is suitable for a prioritized relationship in generalized fractional operator and special functions. The significant contribution of $n$-polynomial $\mathcal{P}$ convex functions is that they take into account the special functions and fractional calculus. We addressed many of the basic characteristics of $n$-polynomial $\mathcal{P}$-convex functions in certain special functions, namely the Euler beta function, the hypergeometric function, 
the modified Bessel function and the $\mathfrak{q}$-digamma function. Additionally, the $n$-polynomial type convexity is used to discuss their roles in fractal analysis and machine learning. For further investigation, taking into account the advanced convexity properties, in the preinvexity context, we may extend this study in inequality theory, quantum calculus, machine learning, robotics, weather forecasting and optimizations, which are promising areas that invite potential investigations.

\author{
Acknowledgements \\ The authors would like to express their sincere thanks to the support of National Natural Science Foundation of China. \\ Funding \\ This work was supported by the National Natural Science Foundation of China (Grant Nos. 11401192, 61673169, \\ 11971142) \\ Availability of data and materials \\ Not applicable. \\ Competing interests \\ The authors declare that they have no competing interests. \\ Authors' contributions \\ All authors contributed equally to the writing of this paper. All authors read and approved the final manuscript.

\section{Author details} \\ ${ }^{1}$ School of Science, Hunan City University, Yiyang, China. '2Department of Mathematics, Government College University, \\ Faisalabad, Pakistan. ${ }^{3}$ Department of Mathematics, COMSATS University Islamabad, Islamabad, Pakistan. ${ }^{4}$ Division of \\ Applied Mathematics, Thu Dau Mot University, Thu Dau Mot City, Binh Duong Province, Vietnam. ${ }^{5}$ Department of \\ Mathematics, Huzhou University, Huzhou, China. ${ }^{6}$ Hunan Provincial Key Laboratory of Mathematical Modeling and \\ Analysis in Engineering, Changsha University of Science \& Technology, Changsha, China.
}

\title{
Publisher's Note
}

Springer Nature remains neutral with regard to jurisdictional claims in published maps and institutional affiliations.

Received: 2 July 2020 Accepted: 23 September 2020 Published online: 02 October 2020

\section{References}

1. Khurshid, Y., Adil Khan, M., Chu, Y.-M.: Conformable fractional integral inequalities for GG- and GA-convex functions. AIMS Math. 5(5), 5012-5030 (2020)

2. Chu, Y.-M., Awan, M.U., Javad, M.Z., Khan, A.W.: Bounds for the remainder in Simpson's inequality via $n$-polynomial convex functions of higher order using Katugampola fractional integrals. J. Math. 2020, Article ID 4189036 (2020)

3. Yan, P.-Y., Li, Q., Chu, Y.-M., Mukhtar, S., Waheed, S.: On some fractional integral inequalities for generalized strongly modified $h$-convex function. AIMS Math. 5(6), 6620-6638 (2020)

4. Ge-JiLe, H., Rashid, S., Noor, M.A., Suhail, A., Chu, Y.-M.: Some unified bounds for exponentially tgs-convex functions governed by conformable fractional operators. AIMS Math. 5(6), 6108-6123 (2020)

5. Kalsoom, H., Idrees, M., Baleanu, D., Chu, Y.-M.: New estimates of $q_{1} q_{2}$-Ostrowski-type inequalities within a class of n-polynomial prevexity of function. J. Funct. Spaces 2020, Article ID 3720798 (2020)

6. Hussain, S., Khalid, J., Chu, Y.-M.: Some generalized fractional integral Simpson's type inequalities with applications. AIMS Math. 5(6), 5859-5883 (2020)

7. Chen, S.-B., Rashid, S., Noor, M.A., Ashraf, R., Chu, Y.-M.: A new approach on fractional calculus and probability function. AIMS Math. 5(6), $7041-7054$ (2020)

8. Shen, J.-M., Rashid, S., Noor, M.A., Ashraf, R., Chu, Y.-M.: Certain novel estimates within fractional calculus theory on time scales. AIMS Math. 5(6), 6073-6086 (2020)

9. Xu, L., Chu, Y.-M., Rashid, S., El-Deeb, A.A., Nisar, K.S.: On new unified bounds for a family of functions with fractional q-calculus theory. J. Funct. Spaces 2020, Article ID 4984612 (2020)

10. Rashid, S., Khalid, A., Rahman, S., Nisar, K.S., Chu, Y.-M.: On new modifications governed by quantum Hahn's integral operator pertaining to fractional calculus. J. Funct. Spaces 2020, Article ID 8262860 (2020)

11. Kalsoom, H., Idrees, M., Kashuri, A., Awan, M.U., Chu, Y.-M.: Some new $\left(p_{1} p_{2}, q_{1} q_{2}\right)$-estimates of Ostrowski-type integral inequalities via n-polynomials s-type convexity. AIMS Math. 5(6), 7122-7144 (2020)

12. Zhou, S.-S., Rashid, S., Jarad, F., Kalsoom, H., Chu, Y.-M.: New estimates considering the generalized proportional Hadamard fractional integral operators. Adv. Differ. Equ. 2020, Article ID 275 (2020)

13. Abdeljawad, T., Rashid, S., Khan, H., Chu, Y.-M.: On new fractional integral inequalities for $p$-convexity within interval-valued functions. Adv. Differ. Equ. 2020, Article ID 330 (2020)

14. Zaheer Ullah, S., Adil Khan, M., Khan, Z.A., Chu, Y.-M.: Coordinate strongly s-convex functions and related results. J. Math. Inequal. 14(3), 829-843 (2020)

15. Podlubny, I.: Fractional Differential Equations. Academic Press, San Diego (1999)

16. Zhao, T.-H., He, Z.-Y., Chu, Y.-M.: On some refinements for inequalities involving zero-balanced hypergeometric function. AIMS Math. 5(6), 6479-6495 (2020) 
17. Wang, M.-K., Chu, Y.-M., Li, Y.-M., Zhang, W.: Asymptotic expansion and bounds for complete elliptic integrals. Math. Inequal. Appl. 23(3), 821-841 (2020)

18. Sun, M.-B., Chu, Y.-M.: Inequalities for the generalized weighted mean values of $g$-convex functions with applications. Rev. R. Acad. Cienc. Exactas Fís. Nat., Ser. A Mat. 114(4), Article ID 172 (2020)

19. Abbas Baloch, I., Mughal, A.A., Chu, Y.-M., Haq, A.U., De La Sen, M.: A variant of Jensen-type inequality and related results for harmonic convex functions. AIMS Math. 5(6), 6404-6418 (2020)

20. Awan, M.U., Talib, S., Noor, M.A., Chu, Y.-M., Noor, K...: Some trapezium-like inequalities involving functions having strongly n-polynomial preinvexity property of higher order. J. Funct. Spaces 2020, Article ID 9154139 (2020)

21. Adil Khan, M., Pečarić, J., Chu, Y.-M.: Refinements of Jensen's and McShane's inequalities with applications. AlMS Math. 5(5), 4931-4945 (2020)

22. Zhao, T.-H., Wang, M.-K., Chu, Y.-M.: A sharp double inequality involving generalized complete elliptic integral of the first kind. AIMS Math. 5(5), 4512-4528 (2020)

23. Huang, X.-F., Wang, M.-K., Shao, H., Zhao, Y.-F., Chu, Y.-M.: Monotonicity properties and bounds for the complete p-elliptic integrals. AIMS Math. 5(6), 7071-7086 (2020)

24. He, Z.-Y., Wang, M.-K., Jiang, Y.-P., Chu, Y.-M.: Bounds for the perimeter of an ellipse in terms of power means. J. Math. Inequal. 14(3), 887-899 (2020)

25. Set, E., Celik, B.: On generalizations related to the left side of Fejer's inequality via fractional integral operator. Miskolc Math. Notes 18(2), 1043-1057 (2017)

26. Ostrowski, A.: Über die Absolutabweichung einer differentiierbaren Funktion von ihrem Integralmittelwert Comment. Math. Helv. 10(1), 226-227 (1937)

27. Işcan, I.: New refinements for integral and sum forms of Hölder inequality. J. Inequal. Appl. 2019, Article 304 (2019)

28. Kadakal, M., İscan, I., Kadakal, H., Bekar, K.: On improvements of some integral inequalities. Researchgate Preprint (2019). https://doi.org/10.13140/RG.2.2.15052.46724

29. Zhang, K.-S. Wan, J.-P.: p-Convex functions and their properties. Pure Appl. Math. 23(1), 130-133 (2007)

30. İscan, I.: Hermit-Hadamard type inequalities for harmonically convex functions. J. Math. Stat. 43(6), 935-942 (2014)

31. Toplu, T., Kadakal, M., İscan, i.: On n-polynomial convexity and some related inequalities. AIMS Math. 5(2), 1304-1318 (2020)

32. Awan, M.U., Akhtar, N., Iftikhar, S., Noor, M.A., Chu, Y.-M.: New Hermite-Hadamard type inequalities for $n$-polynomial harmonically convex functions. J. Inequal. Appl. 2020, Article ID 125 (2020)

33. Katugampola, U.N.: New approach to a generalized fractional integral. Appl. Math. Comput. 218(3), 860-865 (2011)

34. Kilbas, A.A., Srivastava, H.M., Trujillo, J.J.: Theory and Applications of Fractional Differential Equations. Elsevier, Amsterdam (2006)

35. Shen, J.-M., Yang, Z.-H., Qian, W.-M., Zhang, W., Chu, Y.-M.: Sharp rational bounds for the gamma function. Math. Inequal. Appl. 23(3), 843-853 (2020)

36. Toplu, T., Set, E., İscan, I., Maden, S.: Hermite-Hadamard type inequalities for $p$-convex functions via Katugampola fractional integrals. Facta Univ., Ser. Math. Inform. 34(1), 149-164 (2019)

37. Thatsatian, A., Ntouyas, S.K., Tariboon, J.: Some Ostrowski type inequalities for $p$-convex functions via generalized fractional integrals. J. Math. Inequal. 13(2), 467-478 (2019)

38. Zhao, T.-H., Shi, L., Chu, Y.-M.: Convexity and concavity of the modified Bessel functions of the first kind with respect to Hölder means. Rev. R. Acad. Cienc. Exactas Fís. Nat., Ser. A Mat. 114(2), Article ID 96 (2020)

\section{Submit your manuscript to a SpringerOpen ${ }^{\circ}$ journal and benefit from:}

- Convenient online submission

- Rigorous peer review

- Open access: articles freely available online

- High visibility within the field

Retaining the copyright to your article

Submit your next manuscript at $\gg$ springeropen.com 